\title{
Evaluating Coarse-Grained MARTINI Force-Fields for Capturing the Ripple Phase of Lipid Membranes
}

\author{
Pradyumn Sharma, ${ }^{\S}$ Rajat Desikan, ${ }^{\S}$ and K. Ganapathy Ayappa*
}

Cite This: J. Phys. Chem. B 2021, 125, 6587-6599

Read Online

ABSTRACT: Phospholipids, which are an integral component of cell membranes, exhibit a rich variety of lamellar phases modulated by temperature and composition. Molecular dynamics (MD) simulations have greatly enhanced our understanding of phospholipid membranes by capturing experimentally observed phases and phase transitions at molecular resolution. However, the ripple $\left(\mathrm{P}_{\beta^{\prime}}\right)$ membrane phase, observed as an intermediate phase below the main gel-to-liquid crystalline transition with some lipids, has been challenging to capture with MD simulations, both at allatom and coarse-grained (CG) resolutions. Here, with an aggregate $\sim 2.5 \mu$ s all-atom and $\sim 122 \mu$ s CGMD simulations, we systematically assess the ability of six CG MARTINI 1,2-

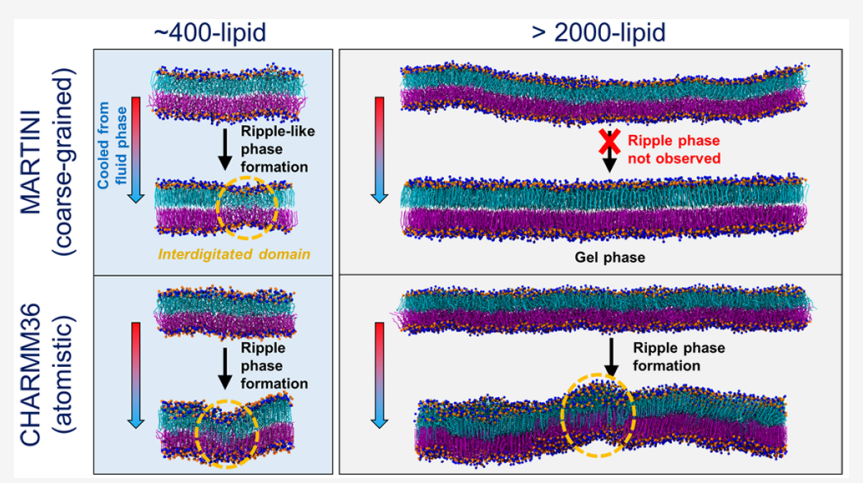
dipalmitoyl-sn-glycero-3-phosphocholine (DPPC) lipid and water force-field (FF) variants, parametrized to capture the DPPC gel and fluid phases, for their ability to capture the $\mathrm{P}_{\beta^{\prime}}$ phase, and compared observations with those from an all-atom FF. Upon cooling from the fluid phase to below the phase transition temperature with smaller (380-lipid) and larger (>2200-lipid) MARTINI and all-atom (CHARMM36 FF) DPPC lipid bilayers, we observed that smaller bilayers with both all-atom and MARTINI FFs sampled interdigitated $\mathrm{P}_{\beta^{\prime}}$ and ripple-like states, respectively. However, while all-atom simulations of the larger DPPC membranes exhibited the formation of the $\mathrm{P}_{\beta^{\prime}}$ phase, MARTINI membranes did not sample interdigitated ripple-like states at larger system sizes. We then demonstrated that the ripple-like states in smaller MARTINI membranes were kinetically trapped structures caused by finite size effects rather than being representative of true $\mathrm{P}_{\beta^{\prime}}$ phases. We showed that a MARTINI FF variant that could capture the tilted $\mathrm{L}_{\beta^{\prime}}$ gel phase, a prerequisite for stabilizing the $\mathrm{P}_{\beta^{\prime}}$ phase, was unable to capture the rippled phase upon cooling. Our study reveals that the current MARTINI FFs (including MARTINI3) may require specific reparametrization of the interaction potentials to stabilize lipid interdigitation, a characteristic of the ripple phase.

\section{INTRODUCTION}

Lamellar lipid membranes, composed of two layers of amphiphilic lipids with solvated hydrophilic "heads" and buried hydrophobic "tails", exhibit a wide variety of thermotropic phases such as crystalline $\left(\mathrm{L}_{\mathrm{c}}\right)$, tilted gel $\left(\mathrm{L}_{\beta^{\prime}}\right)$ or straight gel $\left(\mathrm{L}_{\beta}\right)$, tilted ripple $\left(\mathrm{P}_{\beta^{\prime}}\right)$, and liquid-crystalline $\left(\mathrm{L}_{\alpha}\right) .{ }^{1,2}$ Lipid bilayers are important constituents of biomembranes, which are ubiquitous in living organisms, ${ }^{3}$ and nanoparticle pharmaceuticals such as liposomal drugs. ${ }^{4-6}$ Specifically, lipid membrane phases influence: (i) biological processes at the cellular level such as membrane fusion and budding, ${ }^{7}$ permeation of drugs, ${ }^{8,9}$ effect of anesthetics, ${ }^{10}$ vulnerability to pathogenic toxins, ${ }^{11,12}$ lipid flip-flop, ${ }^{13,14}$ neural signaling, ${ }^{15}$ and the function of membrane proteins ${ }^{3,7}$ and (ii) industrial applications such as the design, storage, and usage of liposomal drugs, ${ }^{4-6,16}$ cosmetic formulations, ${ }^{\text {, and lipid-rich }}$ foods. ${ }^{1}$ Molecular simulations of membranes at various levels of detail have yielded atomistic insights into the rich phase behavior of phospholipid membranes while complementing experimental observations. ${ }^{1,17}$ All-atom molecular dynamics (MD) simulations have successfully captured the low-temper- ature $\mathrm{L}_{\beta^{\prime}}$ or $\mathrm{L}_{\beta}$ gel phases, the liquid-ordered and liquiddisordered phases, and the main gel-to-fluid phase transition in simple lipid bilayers while providing detailed insights into the rate-limiting formation of the melting seed that nucleates the transition. ${ }^{18}$ Similarly, the molecular structure of lipid domains in the experimentally elusive $\mathrm{P}_{\beta^{\prime}}$ phase, which commonly occurs upon cooling membranes from the $\mathrm{L}_{\alpha}$ phase to below the gel-to-fluid transition temperature (pretransition region), was unraveled at atomistic resolution via detailed MD simulations ${ }^{19-21}$ and was found to be consistent with experimental observations.

While these in silico studies illustrate the wide applicability of all-atom lipid force-fields (FFs), all-atom MD simulations

Received: April 12, 2021

Revised: $\quad$ May 19, 2021

Published: June 3, 2021 
Table 1. List of Previous Studies, Which Have Explored the $\mathbf{P}_{\boldsymbol{\beta}^{\prime}}$ Phase Using MD Simulations ${ }^{a}$

\begin{tabular}{|c|c|c|c|c|c|}
\hline lipid & year & resolution & FF & comparison with experiments & citation \\
\hline DPPC & 2005 & united atom & modified Berger lipid & qualitative comparison with DMPC electron density profiles & ref 19 \\
\hline DMPC & 2005 & CG & $\mathrm{DPD}$ & & ref 21 \\
\hline DPPC & 2007 & CG & custom (single-tailed) & qualitative comparison with DPPC electron density maps & ref 20 \\
\hline DMPC, DPPC & 2012 & CG & DPD, MARTINI & $\begin{array}{l}\text { enthalpic signatures of phase transitions from } \mathrm{MD} \text { compared with differential } \\
\text { scanning calorimetry }\end{array}$ & ref 36 \\
\hline $\mathrm{BTMAC}+\mathrm{SA}$ & 2014 & united atom & GROMOS 87, OPLS & & ref 43 \\
\hline DPPC & 2014 & CG & MARTINI & & ref 48 \\
\hline DMPC & 2016 & all-atom & CHARMM36, Lipid14 & & ref 44 \\
\hline DPPC & 2017 & CG & dry MARTINI & & ref 49 \\
\hline DMPC, DPPC & 2018 & all-atom & CHARMM36 & comparison of bilayer properties with X-ray diffraction experiments & ref 46 \\
\hline DPPC & 2018 & all-atom & CHARMM36 & & ref 18 \\
\hline DPPC & 2018 & $\begin{array}{l}\text { all-atom, } \\
\text { CG }\end{array}$ & $\begin{array}{l}\text { CHARMM36, } \\
\text { MARTINI }\end{array}$ & & ref 31 \\
\hline DPPC & 2019 & all-atom & CHARMM36 & & ref 45 \\
\hline BTMAC + SA & 2020 & $\begin{array}{l}\text { all-atom, } \\
\text { CG }\end{array}$ & $\begin{array}{l}\text { CHARMM36, } \\
\text { MARTINI }\end{array}$ & & ref 25 \\
\hline
\end{tabular}

${ }^{a}$ Abbreviations: DPD—dissipative particle dynamics; DPPC-1,2-dipalmitoyl-sn-glycero-3-phosphocholine; DMPC-1,2-dimyristoyl-sn-glycero3-phosphocholine; BTMAC-benzyltrimethylammonium chloride; and SA-stearyl alcohol.

are typically limited by computational bottlenecks to the nanoscale, and complex membrane processes typically require microscale MD simulations. ${ }^{17,22}$ Therefore, coarse-grained (CG) lipid models such as those used in the dissipative particle dynamics (DPD), MARTINI, and other frameworks have been developed. ${ }^{22-26}$ Among the CG models, the MARTINI biomolecular FF has been successfully used to study a variety of complex biological systems outside the purview of all-atom MD simulations. ${ }^{22}$ For example, MARTINI simulations have been used to carry out microsecond simulations of an idealized 20,000-lipid plasma membrane comprising 63 different species of lipids, ${ }^{27}$ a difficult feat with typical all-atom simulations. The MARTINI model is based on a four-to-one mapping scheme, where four non-hydrogen atoms are typically modeled as effective CG interaction sites ("beads"), whose parameters were calibrated by comparison with macroscopic properties such as thermodynamic transfer free energies (details of bead types and FF parameterization in refs $\left.{ }^{22,24,28,33}\right)$. The versatility and computational tractability of CG models such as MARTINI render them as attractive options for capturing the microscopic details of complex membrane phenomena. ${ }^{22,24}$ However, because CG models by definition possess reduced molecular detail $^{28}$ their interaction potentials must be continuously assessed and refined, often by systematic comparison with experiments and/or corresponding detailed all-atom models, ${ }^{25,26,28-33}$ to enable increasingly accurate CGMD simulations.

1,2-Dipalmitoyl-sn-glycero-3-phosphocholine (DPPC) possesses a large headgroup and favorable head-head interactions that stabilize a tilted gel $\left(\mathrm{L}_{\beta^{\prime}}\right)$ phase at lower temperatures, unlike lipids with smaller headgroups such as phosphatidylethanolamine that favor the straight gel $\left(\mathrm{L}_{\beta}\right)$ phase. ${ }^{20,21,34}$ At higher temperatures, DPPC exhibits a main $\mathrm{L}_{\beta^{\prime}}$ to $\mathrm{L}_{\alpha}$ phase transition, with the $\mathrm{P}_{\beta^{\prime}}$ phase occurring at pretransition temperatures. ${ }^{34-40}$ The $\mathrm{P}_{\beta^{\prime}}$ phase has interesting properties such as the influence of thermal history on the symmetry of the ripple domains, ${ }^{20}$ and therefore has been the subject of extensive experimental $35,39-42$ and theoreti$\mathrm{cal}^{18-21,25,31,37,38,43-47}$ studies. In Table 1, we summarize some of the previous studies, where the rippled phase has been observed, including details of the lipid type, resolution (all- atom, united atom, or CG), and FF. Despite extensive in silico investigations, $\mathrm{MD}$ simulations that capture the $\mathrm{P}_{\beta^{\prime}}$ phase have proven to be challenging because the ripple domain structure formation is not only a function of the specific lipid FF (Table $1)$, but also depends on the cooling/heating protocols ${ }^{18,36}$ and system size effects. ${ }^{20,36,45}$ In spite of these challenges, MD simulations of lipid bilayers in the ripple phase have revealed elusive details of molecular organization that were challenging to probe via experiments, such as the asymmetric sawtooth-like molecular organization of lipids in the DPPC ripple domains. ${ }^{19-21}$

While DPPC membranes described by the standard MARTINI FF and its variants have been shown to capture the gel and fluid states, ${ }^{22,31}$ there are conflicting reports of their ability to capture the ripple phase. Some MARTINI studies have reported ripple formation both with and without enhanced sampling, ${ }^{31,48,49}$ while others have reported that MARTINI membranes cannot capture the ripple phase ${ }^{36}$ with the interdigitated ripple-like domains observed upon quasistatic cooling from the $\mathrm{L}_{\alpha}$ phase reported to be artifacts caused by finite size effects. ${ }^{36}$ Therefore, the ability of MARTINI membranes to capture the $\mathrm{P}_{\beta^{\prime}}$ phase still remains an unresolved problem. To systematically address this issue, we assess the ability of commonly employed d $^{18,19,23,29,31,36,45,46,48}$ DPPC bilayers described by six MARTINI lipid FF variants (described subsequently) to capture the interdigitated tilted ripple membrane phase $\left(\mathrm{P}_{\beta^{\prime}}\right)$.

We first establish a standard simulated-annealing-based cooling MD protocol, built on previous studies, ${ }^{18-20,31,45,46}$ which consistently yielded a ripple phase upon cooling from the fluid phase to below the gel-to-fluid-phase transition temperature with all-atom DPPC membranes across multiple simulation replicates. By employing the same protocol with MARTINI DPPC membranes, and by fixing other important variables such as initial configurations $\left(\mathrm{L}_{\alpha}\right.$ phase $)$, system sizes, and cooling rates, we checked whether the MARTINI membranes yielded stable ripple phase structures, similar to those observed in all-atom simulations. Six MARTINI lipid and water FF combinations were tested: (1) standard MARTINI version 2.2 lipid FF with normal water ("MARTINI2n") ${ }^{23}$ or polarizable water ${ }^{50}$ ("MARTINI2p") with embedded charges and explicit charge screening for better 
Table 2. Summary of All-Atom and MARTINI Simulations Performed in This Study

\begin{tabular}{|c|c|c|c|c|c|c|c|c|}
\hline forcefield & $\begin{array}{l}\text { time } \\
(\mu \mathrm{s})^{a}\end{array}$ & $\begin{array}{l}\text { number of } \\
\text { replicas }\end{array}$ & $\begin{array}{c}\text { system size } \\
\left(L_{x} \times L_{y} \times L_{z} \mathrm{~nm}^{3}\right)\end{array}$ & $\begin{array}{l}\text { number of } \\
\text { lipids }\end{array}$ & $\begin{array}{l}\text { number of } \\
\text { particles }\end{array}$ & $\begin{array}{l}\text { type of } \mathrm{MD} \\
\text { simulation }^{c}\end{array}$ & $\begin{array}{l}\text { temperature } \\
(\mathrm{K})\end{array}$ & figures \\
\hline \multicolumn{9}{|c|}{ Small Membrane Simulations } \\
\hline CHARMM36 & 0.10 & 1 & $15.7 \times 7.8 \times 8.4$ & 368 & 103,040 & $\mathrm{EQMD}^{b}$ & 350 & $1, \mathrm{~S} 1$ \\
\hline CHARMM36 & 0.36 & 2 & $13.4 \times 6.7 \times 10.6$ & 368 & 103,040 & SAMD & $350-290$ & \\
\hline CHARMM36 & 0.20 & 2 & $13.4 \times 6.7 \times 10.6$ & 368 & 103,040 & EQMD & 290 & \\
\hline MARTINI2n & 0.10 & 1 & $15.8 \times 7.9 \times 9.6$ & 380 & 9626 & $\mathrm{EQMD}^{b}$ & 350 & 2 \\
\hline MARTINI2On & 0.10 & 1 & $15.8 \times 7.9 \times 9.6$ & 380 & 9620 & $\mathrm{EQMD}^{b}$ & 350 & \\
\hline MARTINI3a & 0.10 & 1 & $16.1 \times 8.0 \times 8.8$ & 380 & 9626 & $\mathrm{EQMD}^{b}$ & 350 & \\
\hline MARTINI3b & 0.10 & 1 & $15.9 \times 7.9 \times 9.1$ & 380 & 9626 & $\mathrm{EQMD}^{b}$ & 350 & \\
\hline MARTINI2p & 0.10 & 1 & $16.0 \times 8.0 \times 9.0$ & 380 & 19,761 & $\mathrm{EQMD}^{b}$ & 350 & \\
\hline MARTINI2Op & 0.10 & 1 & $15.5 \times 7.8 \times 9.5$ & 380 & 19,764 & $\mathrm{EQMD}^{b}$ & 350 & \\
\hline MARTINI2n & 0.42 & 10 & $14.2 \times 7.1 \times 11.1$ & 380 & 9626 & SAMD & $350-280$ & $\mathrm{~S} 2$ \\
\hline MARTINI2On & 0.42 & 10 & $13.9 \times 7.0 \times 11.5$ & 380 & 9620 & SAMD & $350-280$ & S3 \\
\hline MARTINI3a & 0.42 & 10 & $14.1 \times 7.1 \times 10.5$ & 380 & 9626 & SAMD & $350-280$ & S4 \\
\hline MARTINI3b & 0.42 & 10 & $14.2 \times 7.1 \times 10.5$ & 380 & 9626 & SAMD & $350-280$ & S5 \\
\hline MARTINI2p & 0.42 & 10 & $14.4 \times 7.2 \times 10.2$ & 380 & 19,761 & SAMD & $350-280$ & S6 \\
\hline MARTINI2Op & 0.42 & 10 & $13.8 \times 6.9 \times 10.9$ & 380 & 19,764 & SAMD & $350-280$ & S7 \\
\hline MARTINI2n & 5 & $2^{d}$ & $14.3 \times 7.1 \times 11.0$ & 380 & 9626 & EQMD & 280 & 2,6 \\
\hline MARTINI2On & 5 & $3^{e}$ & $13.9 \times 7.0 \times 11.4$ & 380 & 9620 & EQMD & 280 & $2,6, S 13$ \\
\hline MARTINI3a & 5 & $1^{f}$ & $14.1 \times 7.1 \times 10.5$ & 380 & 9626 & EQMD & 280 & 2 \\
\hline MARTINI3b & 5 & $1^{g}$ & $14.0 \times 7.0 \times 10.7$ & 380 & 9626 & EQMD & 280 & $\mathrm{~S} 5 \mathrm{~b}$ \\
\hline MARTINI2p & 5 & $1^{h}$ & $14.3 \times 7.2 \times 10.2$ & 380 & 19,761 & EQMD & 280 & 2 \\
\hline MARTINI2Op & 5 & $1^{i}$ & $13.8 \times 6.9 \times 10.9$ & 380 & 19,764 & EQMD & 280 & 2 \\
\hline \multicolumn{9}{|c|}{ Large Membrane Simulations } \\
\hline CHARMM36 & 0.02 & 1 & $46.4 \times 15.4 \times 16.1$ & 2208 & $1,135,872$ & $\mathrm{EQMD}^{b}$ & 350 & $3, S 8$ \\
\hline CHARMM36 & 0.36 & 2 & $40.3 \times 13.4 \times 19.9$ & 2208 & $1,135,872$ & SAMD & $350-290$ & \\
\hline CHARMM36 & 0.20 & 2 & $40.4 \times 13.5 \times 19.8$ & 2208 & $1,135,872$ & EQMD & 290 & \\
\hline MARTINI2n & 0.10 & 2 & $46.5 \times 15.5 \times 20.6$ & 2204 & 112,719 & $\mathrm{EQMD}^{b}$ & 350 & 4 \\
\hline MARTINI2On & 0.10 & 2 & $46.3 \times 15.4 \times 20.8$ & 2204 & 112,719 & $\mathrm{EQMD}^{b}$ & 350 & \\
\hline MARTINI3a & 0.10 & 2 & $46.6 \times 15.5 \times 19.1$ & 2204 & 112,717 & $\mathrm{EQMD}^{b}$ & 350 & \\
\hline MARTINI3b & 0.10 & 2 & $47.1 \times 15.7 \times 18.8$ & 2204 & 112,717 & $\mathrm{EQMD}^{b}$ & 350 & \\
\hline MARTINI2n & 0.42 & 2 & $39.9 \times 13.3 \times 26.0$ & 2204 & 112,719 & SAMD & $350-280$ & 4, $S 10$ \\
\hline MARTINI2On & 0.42 & 2 & $39.9 \times 13.1 \times 26.6$ & 2204 & 112,719 & SAMD & $350-280$ & \\
\hline MARTINI3a & 0.42 & 2 & $39.3 \times 13.1 \times 24.9$ & 2204 & 112,717 & SAMD & $350-280$ & \\
\hline MARTINI3b & 0.42 & 2 & $41.3 \times 13.8 \times 22.7$ & 2204 & 112,717 & SAMD & $350-280$ & \\
\hline MARTINI2n & 3 & 1 & $39.6 \times 13.2 \times 12.7$ & 2280 & 57,756 & EQMD & 280 & $5, \mathrm{~S} 12$ \\
\hline MARTINI2On & $5,8,10$ & 3 & $39.7 \times 13.2 \times 12.6$ & 2280 & 57,720 & EQMD & 280 & $\begin{array}{l}\text { 5, 6, S12, } \\
\text { S13 }\end{array}$ \\
\hline MARTINI3a & 10 & 1 & $39.8 \times 13.3 \times 11.9$ & 2280 & 57,756 & EQMD & 280 & $5, \mathrm{~S} 12$ \\
\hline MARTINI3b & 10 & 1 & $40.0 \times 13.3 \times 11.8$ & 2280 & 57,756 & EQMD & 280 & S12 \\
\hline MARTINI2On & 0.10 & $1^{j}$ & $46.5 \times 46.5 \times 20.2$ & 6728 & 333,604 & $\mathrm{EQMD}^{b}$ & 350 & S11 \\
\hline MARTINI2On & 0.42 & $2^{j}$ & $39.5 \times 39.5 \times 26.0$ & 6728 & 333,604 & SAMD & $350-280$ & $\mathrm{~S} 11$ \\
\hline
\end{tabular}

${ }^{a}$ Simulation times from MARTINI CGMD were not scaled. Total simulation time in this study: $\sim 2.5 \mu$ s (all-atom MD) and $\sim 122 \mu$ s (MARTINI CGMD). ${ }^{b}$ Initial simulations to equilibrate starting configurations. ${ }^{c}$ EQMD-equilibrium $\mathrm{MD}$ simulations in the $\mathrm{NPT}$ ensemble; SAMDsimulated annealing MD simulations at constant pressure. ${ }^{d}$ Replicates with the "best" ripple-like (Repl. 1 in Figure S2) and straight gel (Repl. 7 in Figure S2) phases were further equilibrated for 5000 ns. ${ }^{e}$ Replicates with the "best" ripple-like (Repl. 5 in Figure S3), tilted ripple-like (Repl. 7 in

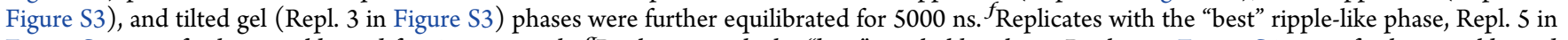
Figure S4, were further equilibrated for 5000 ns each. ${ }^{g}$ Replicates with the "best" ripple-like phase, Repl. 9 in Figure S5, were further equilibrated for 5000 ns each. ${ }^{h}$ Replicates with the "best” ripple-like phase, Repl. 5 in Figure S6, were further equilibrated for 5000 ns each. ${ }^{i}$ Replicates with the "best” ripple-like phase, Repl. 10 in Figure S7, were further equilibrated for 5000 ns each. ${ }^{j}$ Square membrane patch; all others are rectangular membrane patches.

electrostatics, $^{22}$ (2) optimized MARTINI FF for DPPC-has refined bond parameters for better reproduction of DPPC membrane phase behavior and can capture the tilted $\mathrm{L}_{\beta^{\prime}}$ gel phase-with either normal ("MARTINI2On") or polarizable ("MARTINI2Op") water, and, (3) the recently released MARTINI3 alpha ("MARTINI3a") 33 and the slightly different previous interim version MARTINI3 beta (version 3.0.b.3.2; "MARTINI3b"), 51 with their in-built water models. The next generation MARTINI3 FFs include new parameterization of charged beads as well as small and tiny beads involved in aromatic groups, improved bead interactions, and extensive benchmarking, and MARTINI3a is poised to replace MARTINI2 as the default choice of MARTINI FF. ${ }^{33,51}$ For each FF variant, 10 independent replicates of simulated cooling were performed, ensuring sufficient sampling for robust statistical estimates. ${ }^{18,52}$ We observed that smaller (380-lipid) MARTINI DPPC bilayers sampled interdigitated ripple-like states that were not observed with larger DPPC membranes 
a
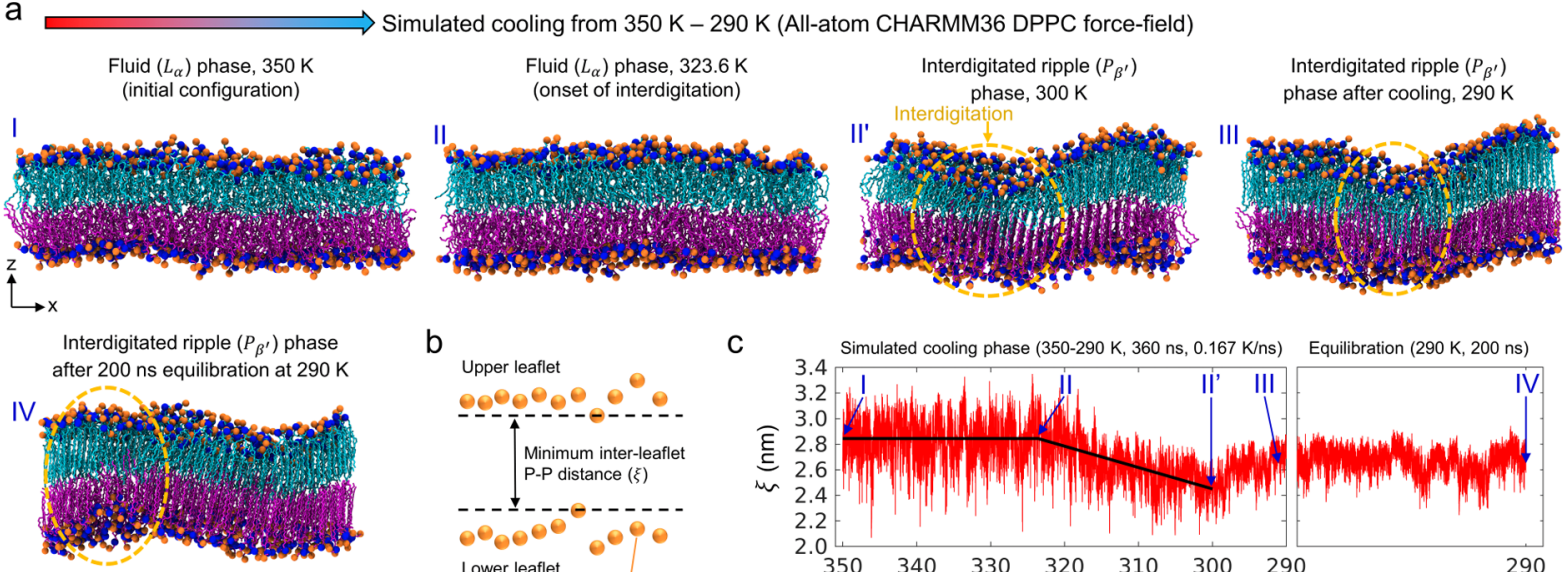

b
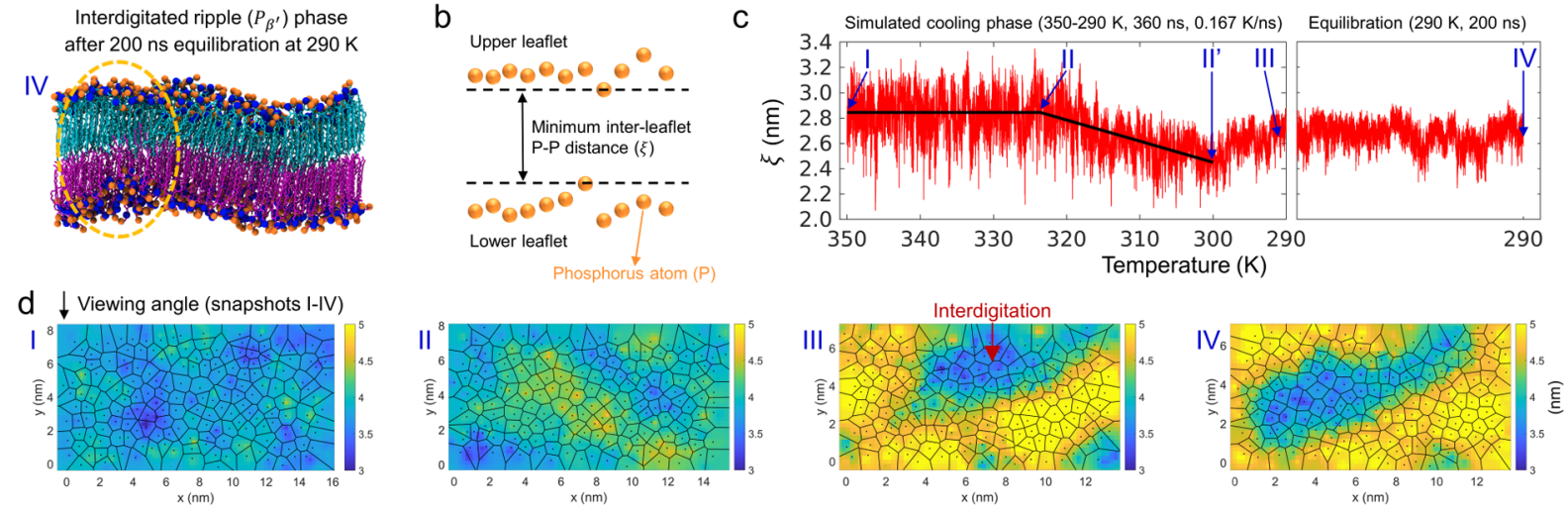

Figure 1. All-atom $\mathrm{L}_{\alpha}$ DPPC membranes consistently form ripples upon cooling. (a) MD snapshots (coloring described in Methods) during the cooling of a 368 -lipid, all-atom, DPPC membrane from 350 to $290 \mathrm{~K}$. Time points: I-initial equilibrated $\mathrm{L}_{\alpha}$ phase membrane at $350 \mathrm{~K}$ before the start of cooling, II - start of interdigitation, II' - time at which the average interleaflet distance in the time series is at a minimum (300 K), III-end of simulated cooling $(290 \mathrm{~K})$, and IV-end of equilibration MD at constant temperature $(290 \mathrm{~K})$. Interdigitated domains are highlighted by yellow-dashed circles. (b) Schematic illustrating the minimum interleaflet $\mathrm{P}-\mathrm{P}$ distance, $\xi$, which is used as an order parameter to track leaflet interdigitation (eq 1). (c) Time trace (red) and biphasic linear fits (black) of $\xi$ in cooling simulations of atomistic DPPC membranes. (d) Voronoi diagram from the $x-y$ coordinates of the upper leaflet $\mathrm{P}$ atoms depicting the area per head group of individual lipids is superimposed on the thickness modulations of membranes at time points I-IV, corresponding to the MD snapshots in (a) (viewing angle pictorially depicted).

(>2200-lipid). The ripple-like states in these smaller MARTINI DPPC membranes were demonstrated to be kinetically trapped structures caused due to finite size effects, rather than being representative of a true ripple phase. However, all-atom simulations of both the smaller and larger DPPC membranes exhibited the formation of the $\mathrm{P}_{\beta^{\prime}}$ phase, similar to previous studies. Thus, our observations assess the ability of MARTINI lipid FFs to capture the ripple phase and serve as a guide for future CG lipid FF development.

\section{METHODS}

All-Atom MD Simulations of DPPC Membranes. The MD protocols employed in this study for simulating DPPC membranes in the gel, ripple, and fluid phases are briefly described below. All the simulations performed in this study are summarized in Table 2.

For the small membrane atomistic simulations, two chargeneutral, solvated, fluid phase $\left(\mathrm{L}_{\alpha}\right)$, 368-lipid membrane configurations at $350 \mathrm{~K}$, with similar spatial coordinates but different starting velocities, were set up using CHARMM$\mathrm{GUI}^{53-55}$ as independent replicates (Figures 1 and S1). The size of the lipid patches was approximately $15.2 \mathrm{~nm}(x$-axis $) \times$ $7.6 \mathrm{~nm}(y$-axis $) \times 9.4 \mathrm{~nm}(z$-axis $)$, with the characteristic dimension of the simulation box in the membrane plane in the range of the periodicity of $12-16 \mathrm{~nm}$ corresponding to previously observed ripple profiles in DPPC membranes. 19,56 This was important because the quality of the observed ripples was found to depend on the characteristic box dimension along the ripple formation. ${ }^{20}$ Systems whose characteristic box length in the membrane plane were noninteger multiples of ripple periodicity resulted in frustrated and interconnected structures, unlike the clean ripples observed in systems with integer multiple box lengths. ${ }^{20}$ Each system contained $>10^{5}$ atoms. The water/lipid ratio was set at 50:1, ensuring sufficient hydration normal to the membrane plane $(z)$, so that the systems could be approximated as isolated lipid bilayers along the $z$-axis.

Atomistic simulations were performed using the CHARMM36 FF ${ }^{44,57,58}$ implementation (March 2019 version from http://mackerell.umaryland.edu/charmm_ff.shtml) in Gromacs (version 2018.6), 59 according to the currently advocated best practices for lipid bilayer simulations ${ }^{17,60-62}$ (see refs 30 and 44 for a brief discussion of all-atom and united atom lipid FFs.). A leapfrog integrator (a 2 fs integration time step) was used along with Verlet buffered lists and threedimensional periodic boundary conditions. Electrostatic interactions were computed with the particle mesh Ewald algorithm, ${ }^{63}$ and van der Waal interactions were smoothly switched to zero between 1.0 and $1.2 \mathrm{~nm}$. Covalent bonds involving hydrogen atoms were constrained using the LINCS algorithm. ${ }^{64}$ Simulations were performed in the NPT ensemble with semi-isotropic pressure coupling along the lateral bilayer plane, by employing the stochastic velocity-rescaling thermostat $^{65}$ for temperature control, and the Parrinello-Rahman barostat $^{66}$ for pressure control, with coupling constants of 0.1 and $2.0 \mathrm{ps}$, respectively. The isothermal compressibilities for the barostat were $\kappa_{X Y}=\kappa_{Z}=4.5 \times 10^{-5} \mathrm{bar}^{-1}$. After energy minimization and equilibration $(100 \mathrm{~ns})$ of the initial configurations in the $\mathrm{L}_{\alpha}$ phase $(350 \mathrm{~K})$ according to standard CHARMM-GUI protocols, ${ }^{54}$ the systemic temperatures of the replicates were continuously reduced from 350 to $290 \mathrm{~K}$ at a rate of $1 \mathrm{~K}$ per $6 \mathrm{~ns}$, or $-0.167 \mathrm{~K} / \mathrm{ns}$, by employing the stochastic simulated annealing MD (SAMD) algorithm implemented in Gromacs ${ }^{59}$ (360 ns per SAMD replicate). 
a

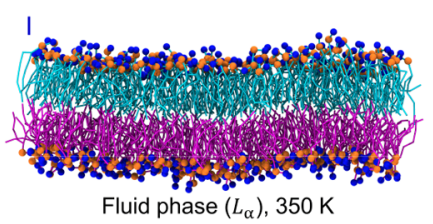

IV
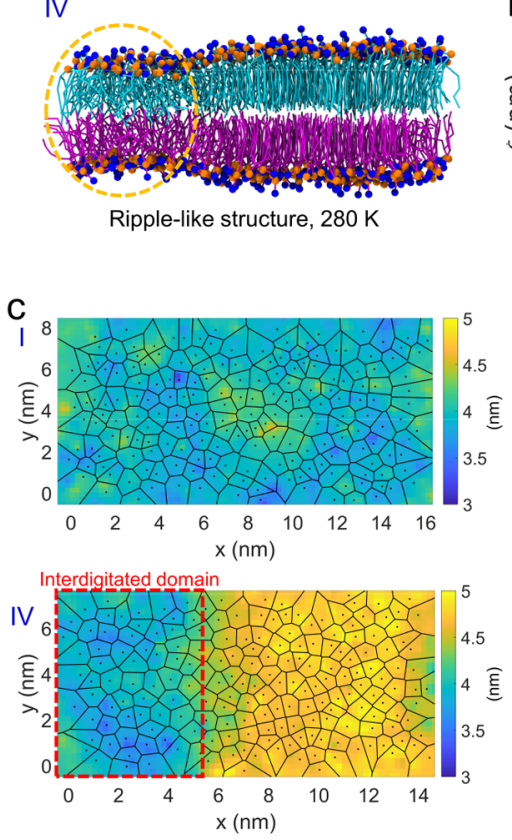

MARTINI2n - 7/10 replicates form an interdigitated 'ripple-like' structure

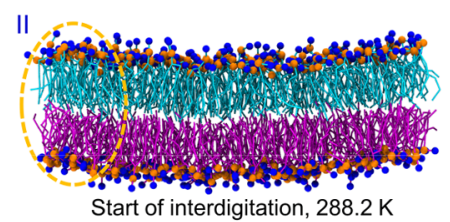

b

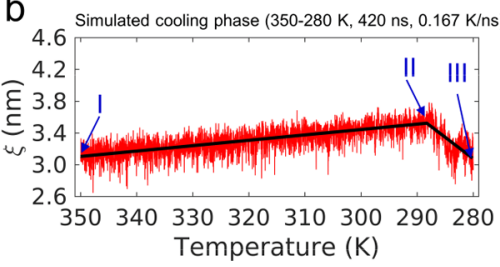

d MARTINI2On $-7 / 10$ replicates

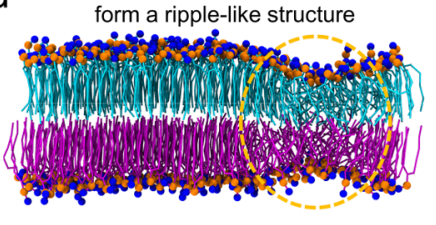

f MARTINI $2 p-1 / 10$ replicates

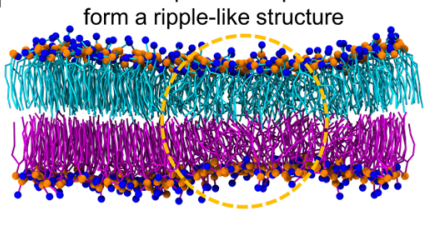

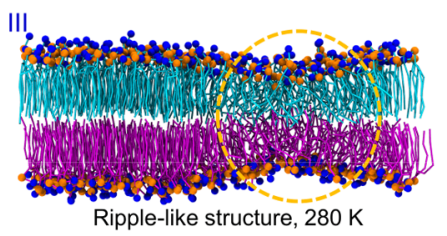

Ripple-like structure, $280 \mathrm{~K}$

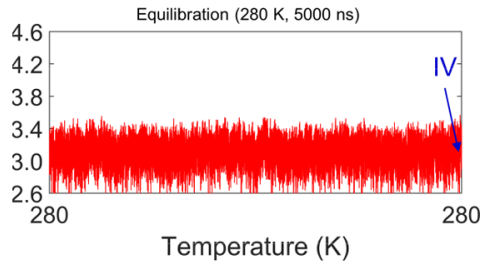

e MARTINI3a $-7 / 10$ replicates form a ripple-like structure
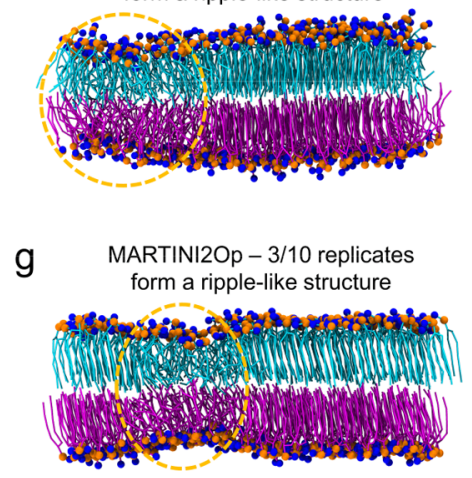

Figure 2. Smaller MARTINI membranes form a ripple-like interdigitated phase upon cooling. (a) Similar to all-atom MD (Figure 1), snapshots from time points I-IV from the simulated cooling of a MARTINI2n membrane replicate from 350 to $280 \mathrm{~K}$ are shown (replicate 1 from Figure S2). Interdigitated regions are highlighted by a yellow-dashed circle. (b) Minimum interleaflet $\mathrm{P}-\mathrm{P}$ distance with time in the 420 ns cooling and $5000 \mathrm{~ns}$ constant temperature equilibration phases. (c) Voronoi-thickness maps contrasting the initial fluid membrane and the final interdigitated ripple-like membrane (time points I and IV, respectively). Cooling of 7/10 independent MARTINI2n MD replicates resulted in the formation of a ripple-like phase (Figure S2). Other MARTINI FF variants: (d) MARTINI2On (replicate 5 from Figure S3), (e) MARTINI3a (replicate 5 from Figure S4; MARTINI3b in Figure S5), (f) MARTINI2p (replicate 5 from Figure S6; formed a ripple-like state during EQMD and not SAMD), and (g) MARTINI2Op (replicate 10 from Figure S7) show the formation of ripple-like phases in 7, 7, 1, and 3 out of 10 replicates each, respectively. These snapshots correspond to time point IV.

We chose to cool slightly beyond the pretransition temperature of $300-310 \mathrm{~K}$ at which the $\mathrm{P}_{\beta^{\prime}}$ phase is typically observed, ${ }^{18,34,36-38}$ because supercooling is often required in kinetic MD simulations for eliciting a phase transition. ${ }^{18,67,68}$ The resulting cooled bilayers at $290 \mathrm{~K}$ were further equilibrated at a constant temperature of $290 \mathrm{~K}$ for $200 \mathrm{~ns}$ for thermal and structural stability.

To test for finite size effects and to compare with MARTINI simulations (described next), two large 2208-lipid membranes measuring $47.0 \mathrm{~nm} \times 15.7 \mathrm{~nm} \times 15.0 \mathrm{~nm}$ and possessing a high water/lipid ratio of 128:1 ( $>10^{6}$ atoms total; Table 2) were equilibrated in the $\mathrm{L}_{\alpha}$ phase at $350 \mathrm{~K}$. Subsequently, both replicates were cooled to $290 \mathrm{~K}$ at a rate of $-0.167 \mathrm{~K} / \mathrm{ns}$ (chosen based on ref 18) and then equilibrated at $290 \mathrm{~K}$ for an additional 200 ns.

MARTINI Simulations of DPPC Membranes. 380-lipid MARTINI DPPC membranes of size $15 \mathrm{~nm} \times 7.5 \mathrm{~nm} \times 10$ nm were prepared using the "INSANE" python module. ${ }^{69}$ The water-bead/lipid ratio was $\sim 13.3: 1$. Because each water bead is equivalent to four water molecules, this corresponds to a water/lipid ratio of $\sim 53: 1$, similar to the small all-atom membranes. As described in the Introduction, six MARTINI FF variants were used in this study: MARTINI2n, ${ }^{23}$
MARTINI2p, ${ }^{50}$ MARTINI2On, ${ }^{31}$ MARTINI2Op, ${ }^{31,50}$ MARTINI3 $a,{ }^{33}$ and MARTINI3b. ${ }^{51}$ MARTINI2n and MARTINI2On systems also contained antifreeze solvent beads ${ }^{23}$ to prevent any artifactual freezing of MARTINI water beads.

Input CGMD parameters were fixed based on previously reported parameter settings for improved performance. ${ }^{70}$ All CGMD simulations were performed with a 20 fs timestep in the NPT ensemble by employing the stochastic velocityrescaling thermostat ${ }^{65}$ (a coupling constant of $1.0 \mathrm{ps}$ ) and the Parrinello-Rahman semi-isotropic barostat ${ }^{66}$ (a coupling constant of $12.0 \mathrm{ps}$ ). The isothermal compressibilities for the barostat were $\kappa_{X Y}=\kappa_{Z}=3 \times 10^{-4} \mathrm{bar}^{-1}$. Electrostatics were treated with the reaction-field algorithm ${ }^{71}$ with a cutoff of 1.1 $\mathrm{nm}$, a relative dielectric constant of 2.5 and 15 for systems with and without polarizable water, respectively, and the relative dielectric constant of the reaction field set to infinity. The van der Waal forces were computed with a cutoff of $1.1 \mathrm{~nm}$. Post energy minimization, six CGMD membranes, each described by a MARTINI FF variant, were equilibrated in the $\mathrm{L}_{\alpha}$ phase at $350 \mathrm{~K}$ for $100 \mathrm{~ns}$, followed by simulated cooling from 350 to $280 \mathrm{~K}$ at $-0.167 \mathrm{~K} / \mathrm{ns}$ (420 ns per SAMD replicate). For each FF, 10 independent SAMD replicates were simulated for ensuring robustness of observations, ${ }^{52}$ resulting in a total of 60 
CG-SAMD simulations (Figures 2, S2-S7, Table 2). The replicates that yielded the best ripple-like phases were further equilibrated for $1-5 \mu \mathrm{s}$ at $280 \mathrm{~K}$ to ensure that the ripples had attained thermal and structural equilibrium (Figures 2 and $\mathrm{S} 5 \mathrm{~b})$.

To test for finite-size effects, many large MARTINI DPPC bilayer configurations, with both rectangular and square geometries, were constructed and simulated (Table 2).

Analysis and Visualization. MD snapshots are illustrated using the visual molecular dynamics 1.9.3 (VMD) ${ }^{72}$ software. The DPPC tails in the upper and lower leaflets of both allatom and MARTINI membranes are consistently colored cyan and magenta, respectively. In all-atom membranes, phosphorus atoms (P) are colored orange, and nitrogen atoms in the choline group are colored blue. Similarly, in MARTINI membranes, the phosphate bead is colored orange and the choline bead is colored blue. The minimum interleaflet $\mathrm{P}-\mathrm{P}$ distance $(\xi)$, which is an order parameter to track leaflet interdigitation (Figure 1b), was computed using the inbuilt "gmx mindist" tool in Gromacs. The $\xi$ values from the fluid and interdigitated regimes are fit to a four-parameter piecewise-linear function (eq 1 ) to accurately estimate the time of the onset of membrane interdigitation, $\tau$.

$$
\begin{aligned}
\xi= & \left(m_{1} t+c\right)(1-H(t-\tau))+\left(m_{2} t+\left(m_{1}-m_{2}\right) \tau\right. \\
& +c) H(t-\tau)
\end{aligned}
$$

Here, $m_{1}$ and $m_{2}$ are the slopes of the two lines corresponding to the fluid and interdigitated regimes (when it occurs), respectively, $c$ is the intercept of the fluid-phase line (the intercept of the other line is $\left(m_{1}-m_{2}\right) \tau+c$ from the point of intersection), and $H(t-\tau)$ is the Heaviside function, which is zero for $t<\tau$ and 1 for $t \geq \tau$. If interdigitation occurs, $m_{2}<0$, else $m_{2}>0$ (illustrated in Figure S2). The fitting was performed using the "lsqcurvefit" function (optimization toolbox) in conjunction with "multistart" (global optimization toolbox) implemented in MATLAB 2018a (https://www. mathworks.com). All other plots are also created with MATLAB. The combined Voronoi-thickness modulation heatmaps are plotted using the "voronoi" MATLAB function on the lipid phosphorus atom $x, y$ coordinates (upper leaflet) from the corresponding MD snapshot. The membrane thickness ${ }^{43}$ is computed using the "Membplugin" 1.1 tool $^{73}$ in VMD and visualized using MATLAB. Similar to a previous study, ${ }^{74}$ lipid tilt angles from MARTINI membranes $\left(\mathrm{L}_{\beta}\right.$ vs $\mathrm{L}_{\beta^{\prime}}$ ) were computed from the last $10 \mathrm{~ns}$ of the respective $\mathrm{MD}$ trajectories, using the inbuilt "gmx gangle" tool in Gromacs. The lipid tilt was defined as the angle between the lipid vector and the leaflet normal, with the lipid vector defined as the vector joining the center of mass of the terminal tail beads with the head phosphate bead.

\section{RESULTS AND DISCUSSION}

Ripple Formation in Atomistic Simulations of DPPC Membranes. To robustly capture the ripple phase in all-atom DPPC membranes, well-equilibrated, fluid, 368-lipid DPPC membranes at $350 \mathrm{~K}$ (time point I in Figure 1a,c) were cooled to $290 \mathrm{~K}$, lower than the experimentally observed pretransition temperature of $300-310 \mathrm{~K}^{34,36-38}$ and sufficient to ensure leaflet interdigitation, ${ }^{18,46}$ using the stochastic simulated annealing $\mathrm{MD}$ algorithm, followed by constant temperature equilibration MD (EQMD) in the NPT ensemble at $290 \mathrm{~K}$ to ensure thermal equilibration and structural convergence (details in Methods; two independent MD replicates). Along with the initial state (time point I), three other time points are important in the combined SAMD + EQMD trajectory (Figure la,c) - the fluid membrane configuration, where interdigitation starts (time point II), the fully interdigitated ripple phase membranes at the end of simulated cooling (time point III), and the converged systems at the end of constant temperature equilibration (time point IV). The extent of interdigitation was tracked by the minimum interleaflet $\mathrm{P}-\mathrm{P}$ distance with time ( $\xi$; see the Methods section and eq 1; Figure $1 \mathrm{~b}, \mathrm{c}$ ). The initial value marked the baseline corresponding to the fluid phase $(350 \mathrm{~K}$; time point $\mathrm{I})$, and an abruptly decreasing trend indicated significant interdigitation of lipids from opposite leaflets, with the onset of interdigitation marked by the inflection point (time point II; see the Methods section, Figure S2, and eq 1). By the end of simulated cooling (290 K; time point III), interdigitation was complete and a membrane with an "asymmetric" $1 \mathrm{D}$ ripple was formed, similar in structure to those reported in previous studies, ${ }^{19,20,45,46}$ structurally stable upon further $200 \mathrm{~ns}$ equilibration at $290 \mathrm{~K}$ (time point IV), and with clearly distinct major and minor arms (illustrated in Figure S1).

Voronoi-thickness membrane maps, which are Voronoi diagrams of the upper membrane leaflet superimposed onto $\mathrm{P}-\mathrm{P}$ thickness heatmaps, ${ }^{25,43}$ are shown in Figure $1 \mathrm{~d}$, corresponding to the top views of MD snapshots in Figure 1a. The trends were similar ${ }^{43}$ when the Voronoi diagrams were plotted using the lower leaflets (not shown). Compared to the uniform topography in the fluid phase, the formation of interdigitated (blue) and gel (yellow) domains in the ripple phase can be clearly observed. There is negligible difference in membrane topographies between time points III and IV except for the lateral movement of the membrane across period boundaries, indicating that the ripple phase membrane configuration at the end of simulated cooling is at equilibrium. An interesting observation was that the interdigitated ripple domain (Figure 1d) appeared to be tilted in the lateral plane, whereas ripple domains in previous studies ${ }^{19,20,43}$ were typically parallel to one of the lateral box edges. Previous mesoscopic lipid bilayer simulations ${ }^{21}$ have revealed that at the highest value of the lipid head-head repulsion parameter explored, lower and higher values of which either abrogated or stabilized the ripple phase, respectively, the bilayer formed a laterally tilted ripple domain and a stable $\mathrm{P}_{\beta^{\prime}}$ membrane phase similar to our observations.

Another independent all-atom MD replicate also yielded a similar interdigitated DPPC ripple phase membrane upon cooling (Figure S1b), thus validating the robustness of the above $\mathrm{MD}$ protocol to produce the $\mathrm{P}_{\beta^{\prime}}$ phase from fluid membrane configurations. We next employed this MD cooling protocol to fluid-phase MARTINI membranes and evaluated their ability to capture the ripple phase.

Ripple Formation in Smaller MARTINI Membranes. Fluid-phase, 380-lipid, DPPC MARTINI membranes belonging to six MARTINI FF variants were cooled from 350 to 280 $\mathrm{K}$, and evaluated for their ability to form the interdigitated $\mathrm{P}_{\beta^{\prime}}$ membrane phase (see Introduction and Methods). The lower bound for temperature was set to $280 \mathrm{~K}$, compared to $290 \mathrm{~K}$ for all-atom, because a previous CGMD study showed that ripple-like interdigitated phases in MARTINI DPPC membranes were formed between 280 and $290 \mathrm{~K}^{36}$ The trajectory of an SAMD + EQMD replicate with the MARTINI2n FF is shown with time points I-IV highlighted (Figure $2 \mathrm{a}-\mathrm{c}$ ). The 
constant temperature EQMD phase post cooling was chosen to be $5 \mu$ s for MARTINI (Figure 2b), compared to $200 \mathrm{~ns}$ for the all-atom simulations (Figure 1c). Similar to the time course seen in atomistic DPPC membranes, a majority (70\%), but notably not all, MARTINI2n replicates showed interdigitation of membrane leaflets (time points I-IV on the $\xi(t)$ plot, Voronoi-thickness maps pertaining to $\mathrm{MD}$ snapshots at time points I and IV), thus apparently capturing ripple-like membrane states. This is in accord with previous single replicate studies, where MARTINI membranes of similar sizes captured the ripple phase. ${ }^{31,36,48,49}$ In the rest of the MARTINI2n replicates (30\%), the membranes bypassed the interdigitated ripple-like state and directly froze into $\mathrm{L}_{\beta}$ configurations (Figure S2). This emphasizes that with simulated cooling $\mathrm{MD}$, rippling in MARTINI membranes depended on the initial configuration and starting velocities, and therefore necessitated multiple MD replicates for obtaining robust trends. ${ }^{52}$

MARTINI SAMD simulations showed two differences compared to all-atom SAMD simulations. First, during SAMD, the atomistic DPPC membranes stay in the fluid phase with disordered tails before the onset of interdigitation (time point II in Figure 1a), and therefore $m_{1} \approx 0$ (Figure 1c; eq 1$)$. In contrast, $m_{1}>0$ for the MARTINI DPPC membranes because they exhibit a degree of tail ordering and expansion along the $Z$-axis (compensated by reduced $X-Y$ dimensions) with decreasing temperature before the start of interdigitation (time point II in Figure 2a), compared to the fluid phase at $350 \mathrm{~K}$ (time point I in Figure 2a). Second, the orientation of the interdigitated domains in the MARTINI2n membranes was perpendicular to the $x$-axis (Figure 2c), in contrast with the laterally tilted interdigitated domains seen in all-atom simulations (Figures $1 \mathrm{~d}$ and S1b). Similar domains were observed in a previous MARTINI study. ${ }^{36}$

Formation of ripple-like phases in DPPC membranes described by the other FF variants-MARTINI2On, MARTINI3a, MARTINI3b, MARTINI2p, and MARTINI2Op-is summarized in Figure $2 \mathrm{~d}-\mathrm{g}$ and $\mathrm{S} 5 \mathrm{~b}$ (all MD replicates for each FF variant are displayed in Figures S3-S7). MARTINI2On is similar to MARTINI2n, and showed ripple-like formation in $70 \%$ of the replicates (Figures $2 \mathrm{~d}$ and S3). MARTINI3a, the improved ${ }^{33,51}$ next generation MARTINI3 FF, showed ripple-like formation in $70 \%$ of the replicates, similar to MARTINI2n and MARTINI2On (Figures 2e and S4) (the beta version of MARTINI3-MARTINI3b-showed ripple-like formation in $20 \%$ of the replicates (Figure S5)). The MARTINI FF combinations with polarizable water, MARTINI2p (Figures $2 \mathrm{f}$ and S6) and MARTINI2Op (Figures $2 \mathrm{~g}$ and S7), showed ripple-like states in only 10 and $30 \%$ of replicates, respectively, despite the reparameterization of polarizable water to improve the accuracy of solvent electrostatic effects, ${ }^{50}$ important for capturing macroscopic membrane properties. $^{57,58}$ Our observations with MARTINI2p are to be contrasted with previous single-replicate MARTINI ${ }^{36}$ simulations, where quasistatic cooling of fluid-phase membranes resulted in the formation of the ripple-like domains only with polarizable water. While differences in MD protocols and differences in the initial configurations may explain the disparities, careful future investigations, with systems of varying size, may be necessary to determine the predictive accuracy of polarizable water-lipid interactions.

Testing for Ripple Formation in Large Atomistic and MARTINI DPPC Membranes upon Simulated Cooling. In a previous study, Rodgers et al. ${ }^{36}$ observed the interdigitation of lipids in relatively small 512-lipid MARTINI DPPC membranes upon quasi-equilibrium, stepwise, simulated cooling of fluid membranes from $325 \mathrm{~K}$ to less than $280 \mathrm{~K}$. These small membranes sampled an interdigitated ripple-like phase, similar to our observations (Figure 2). However, when the exercise was repeated with four times larger (2048-lipid) DPPC membranes, the ripple-like phase could not be reproduced. Thus, Rodgers et al. ${ }^{36}$ concluded that the interdigitated ripple-like states in smaller membranes were actually "intermediate" kinetically trapped membrane states rather than a true ripple phase. In contrast to these MARTINI simulations, a large all-atom 3200-lipid DPPC membrane has been shown to form an interdigitated ripple phase, ${ }^{31}$ albeit with a different $\mathrm{MD}$ protocol. Therefore, we next tested ripple formation in large all-atom DPPC membranes with our simulated cooling MD protocol (similar to Figure 1), that would re-establish a benchmark to test for ripple formation and system size effects in large MARTINI membranes.

We set up two 2208-lipid DPPC bilayer configurations, which were six times larger than the smaller atomistic DPPC membranes shown in Figure 1, and larger than the membranes employed by Rodgers et al., ${ }^{36}$ and pre-equilibrated them in the $\mathrm{L}_{\alpha}$ phase at $350 \mathrm{~K}$ (Figure 3a). Subsequently, these membranes were cooled to $290 \mathrm{~K}$ with the same SAMD protocol. One of the all-atom $\mathrm{MD}$ replicates in the fluid phase formed an interdigitated ripple phase membrane by $290 \mathrm{~K}$ (Figure 3b). The other replicate (Figure S8) formed a noninterdigitated "symmetric" ripple ${ }^{20}$ in the larger dimension, which is typically observed upon slow cooling from the fluid phase to approximately the gel-to-fluid transition temperature, ${ }^{20}$ and an asymmetric interdigitated ripple (similar to Figures 1,3 ) in the smaller dimension. Therefore, both smaller and larger allatom DPPC membranes, described by the CHARMM36 FF, sample the thermodynamic ripple phase upon simulated cooling MD without any evidence of finite size artifacts. In contrast to smaller membranes (Figures 1d, S9a, and S1), the Voronoi-thickness map of the first large membrane replicate shows reticulated ripple domains (Figures $3 \mathrm{~b}$ and $59 \mathrm{~b}$ ). Our observations are in accordance with previous studies, ${ }^{31,45}$ where simulated quenching of 1280-lipid and 3200-lipid allatom DPPC membranes to below the main transition temperature also resulted in reticulated ripple domains. Significantly longer runs are probably required to anneal and reorganize these reticulated ripple domains into classically observed periodic ripple domains.

We next tested whether large, fluid, and rectangular 2204lipid MARTINI DPPC membranes, with normal (not polarizable) MARTINI water, also sampled the interdigitated ripple-like phase upon cooling from 350 to $280 \mathrm{~K}$. Two initial configurations each for MARTINI2n, MARTINI2On, MARTINI3a, and MARTINI3b FFs were set up analogous to the large all-atom membranes in Figure 3, and pre-equilibrated at $350 \mathrm{~K}$, followed by cooling to $280 \mathrm{~K}$ (Figures 4 and S10). However, none of the large MARTINI membranes captured a ripple-like phase upon cooling to $280 \mathrm{~K}$. MARTINI2n and MARTINI3a/b membranes directly froze into the $\mathrm{L}_{\beta}$ phase, while the MARTINI2On membranes transitioned to the $\mathrm{L}_{\beta^{\prime}}$ phase (Figure 4b); confirmed by independent MD replicates (Figure S10). To ensure that membrane geometry did not influence these results, we repeated these simulations with large 6728-lipid square MARTINI2On membranes, and these also did not sample ripple-like states (Figure S11). Because 
a
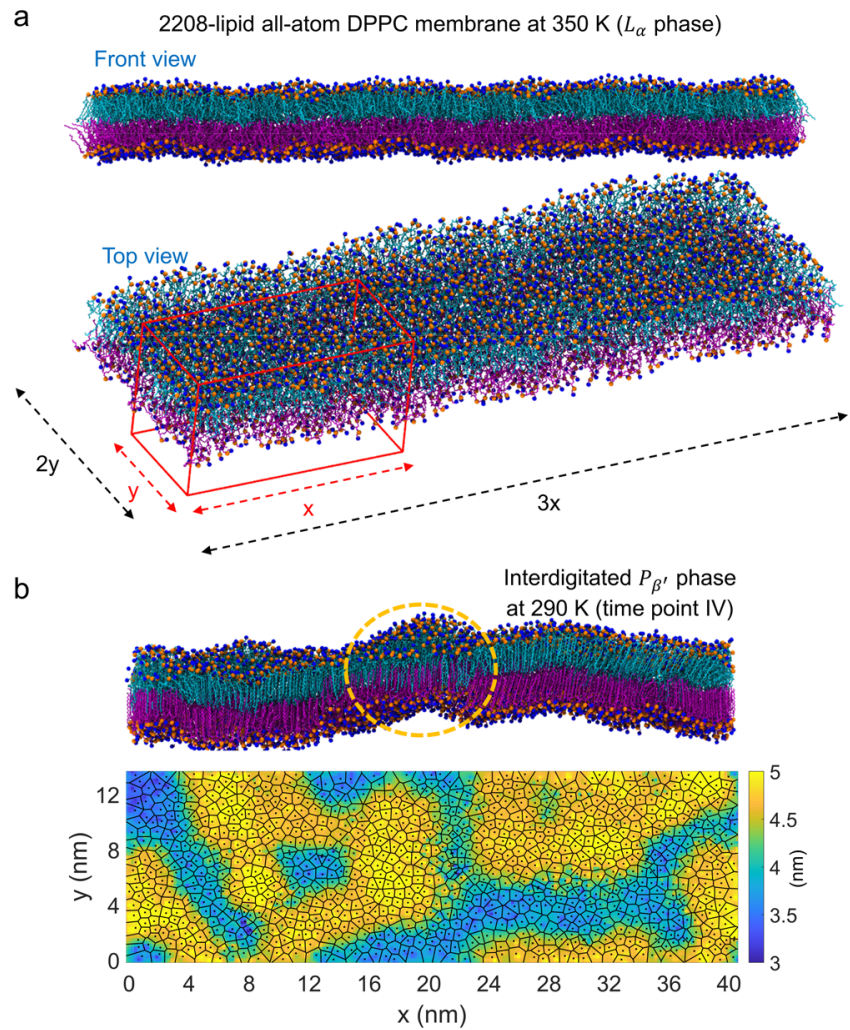

Figure 3. Ripple formation from all-atom MD of a large DPPC membrane. (a) Front and top views of a large 2208-lipid DPPC membrane, which was pre-equilibrated in the fluid phase at $350 \mathrm{~K}$. For comparison, a representative simulation box of a smaller all-atom membrane (Figure 1) is shown in red. The large membrane is six times ( $3 X$ times $2 Y$ ) larger than the smaller membrane. (b) Front view of the large membrane after cooling to $290 \mathrm{~K}$ and EQMD (corresponds to time point IV), and its Voronoi-thickness modulation map (blue represents interdigitated regions) shows the formation of an aperiodic and reticulated interdigitated ripple domain (also see Figures S8 and S9).

only smaller (380-lipid) but not larger (>2204-lipid) DPPC MARTINI membranes captured the interdigitated ripple-like states, in contrast to all-atom membranes, we inferred that the smaller MARTINI ripple-like membranes (Figures 2, S2-S7) represent kinetically trapped states caused by finite box-size effects rather than true ripple states.

Equilibrium MD Simulations of Large MARTINI Membranes Initialized in the Ripple-like Phase Show Loss of Interdigitation. To confirm the aforementioned inference, we employed an orthogonal approach, where multi$\mu \mathrm{s}$ equilibrium simulations of large MARTINI membranes, initialized in periodic interdigitated ripple-like configurations, were performed at $280 \mathrm{~K}$. These large systems were created from the final, interdigitated, ripple-like, and smaller (380lipid) MARTINI membrane configurations after $1 \mu \mathrm{s}$ of constant temperature EQMD at $280 \mathrm{~K}$. The smaller membranes were repeated six times along the lateral bilayer plane across periodic boundaries ( $3 x$ times $2 y$ ), and the initial large 2280-lipid ripple-like membrane configurations thus created are displayed in Figures 5a,c,d and S12d, corresponding to MARTINI2On, MARTINI2n, MARTINI3a, and MARTINI3b, respectively.

The rationale behind performing equilibrium $\mathrm{MD}$ of these large systems was simply to test if the ripple-like states in small
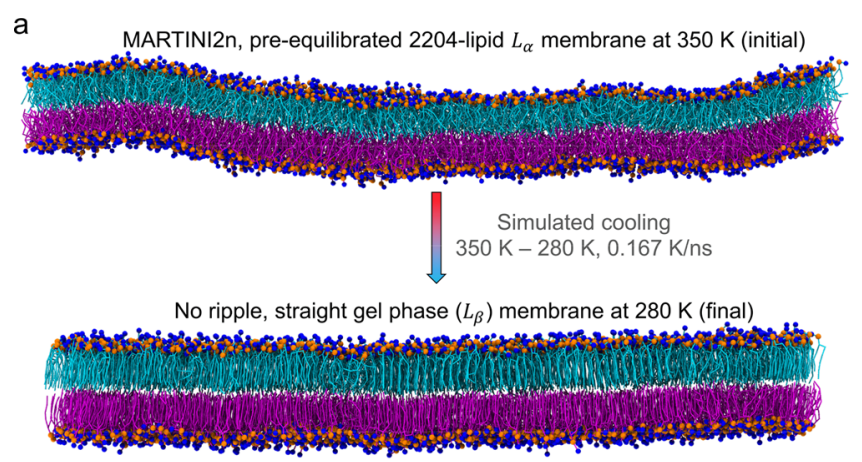

b

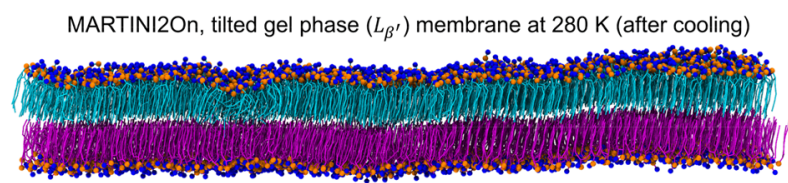

C

MARTINI3a, straight gel phase $\left(L_{\beta}\right)$ membrane at $280 \mathrm{~K}$ (after cooling)

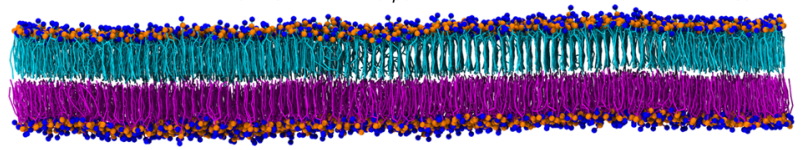

Figure 4. Lack of ripple formation in large MARTINI DPPC membranes. (a) A representative 2204-lipid, rectangular, MARTINI2n DPPC membrane, pre-equilibrated at $350 \mathrm{~K}$ in the $\mathrm{L}_{\alpha}$ phase, was cooled directly into the gel phase at $280 \mathrm{~K}$ without the membrane sampling a ripple-like phase, unlike all-atom systems (see Figure 3). This behavior was also observed with (b) MARTINI2On and (c) MARTINI3a FFs. Independent MD replicates are shown in Figure S10. Two independent simulation replicates, where large 6728-lipid, square MARTINI2On membranes were cooled to $290 \mathrm{~K}$, also showed a similar lack of sampling of a ripple-like phase (Figure S11), suggesting that system geometry did not influence our observations.

systems were stabilized due to the finite size effects. Upon 3$10 \mu$ s of constant temperature NPT-MD at $280 \mathrm{~K}$, we observed that the large, interdigitated MARTINI membranes spontaneously froze into the gel phase, as shown by both MD snapshots (Figures 5 and S12d) and Voronoi-thickness maps (Figure $\mathrm{S} 12 \mathrm{a}-\mathrm{d}$ ), indicating that the initial ripple-like membrane configuration was unfavorable at larger system sizes. This phenomenon was observed for all the four MARTINI FF variants, although the time scale over which interdigitation disappeared leading to formation of the final gel phase configuration varied among the FFs. MARTINI2On showed progressive loss of interdigitation, which was nearly complete by $8 \mu \mathrm{s}$ (Figures 5b and S10a). Longer simulations may have shown a complete loss of interdigitation, but we did not further pursue these computationally intensive MD simulations because the progressive loss of interdigitation with time was apparent (Figures 5a,b and S12a). MARTINI2n showed a rapid and complete loss of interdigitation by $3 \mu \mathrm{s}$ (Figures 5c and S12b), and MARTINI3a/b showed nearcomplete loss of interdigitation by $10 \mu \mathrm{s}$ (Figures $5 \mathrm{~d}$ and S12c,d), similar to MARTINI2On. In contrast, none of the smaller membranes showed any loss of interdigitation upon 5 $\mu$ s of constant temperature EQMD at $280 \mathrm{~K}$ (Figures 2 and $\mathrm{S} 5 \mathrm{~b})$.

Together with observations from the simulated cooling of large fluid membranes, these two orthogonal MD approaches (Figures 4, 5, S10-S12) strongly suggest that MARTINI membranes (with all the tested FFs) do not capture the 
a

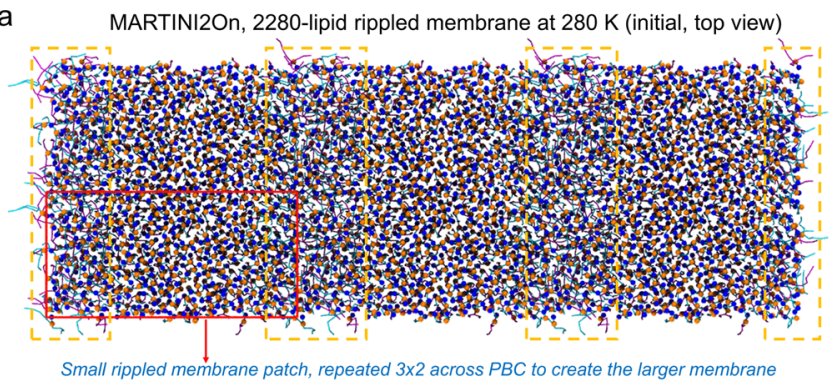

Front view

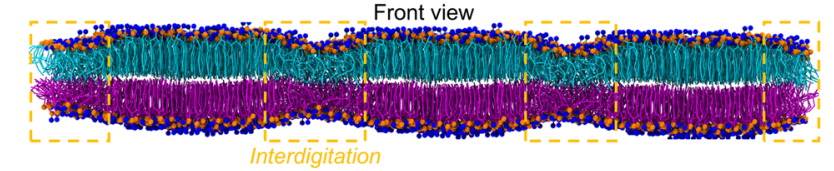

b

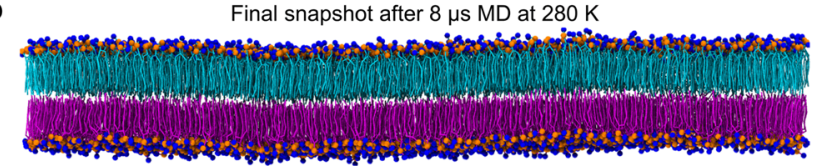

C

MARTINI2n (initial, front view)

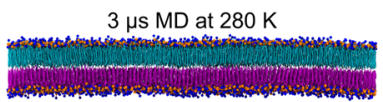

d

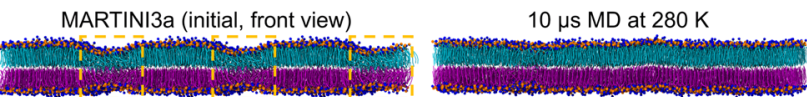

Figure 5. MD simulations of large MARTINI DPPC membranes starting with interdigitated ripple-like states. (a) To test the stability of kinetically trapped domains (yellow-dashed rectangles) at larger system sizes, a 2280-lipid MARTINI2On DPPC membrane configuration with periodic ripples (top and front views) was created by repeating smaller ripple-like membranes (from time point IV, Figure 2d) six times across periodic boundary conditions (thrice in $X$ and twice in $Y$ ). (b) $8 \mu \mathrm{s} \mathrm{MD}$ at $280 \mathrm{~K}$ almost completely abolished the interdigitated ripple-like configuration and led to the establishment of the $\mathrm{L}_{\beta}$ phase (Voronoi-thickness maps in Figure S12a). Similar loss of the ripple-like domains was seen for large MARTINI2n (c) and MARTINI3a (d) membranes (Voronoi-thickness maps in Figure S12b,c).

thermodynamic ripple phase, unlike all-atom membranes. Our systematic study implies that ripple-like phase formation observed in smaller MARTINI membranes was due to kinetic trapping of interdigitated leaflets at small system sizes. The few studies that indeed report MARTINI ripple phase membranes, both with ${ }^{49}$ and without ${ }^{36,48}$ enhanced sampling, are with smaller membranes composed of $390^{49}$ and $512^{36,48}$ lipids, respectively. Therefore, based on our observations, the formation of rippled bilayers in these studies could be attributed to finite size artifacts ${ }^{36}$ until they are validated with simulations at larger system sizes. Thus, MARTINI FF parameters may have to be tuned to better reproduce the lipid membrane phase diagram, and the $\mathrm{P}_{\beta^{\prime}}$ phase in particular. FF approaches that tune the bonded parameters of MARTINI lipids based on comparison with all-atom simulations ${ }^{25,31,36}$ may result in models that better capture the membrane phase behavior.

Optimized MARTINI DPPC FF (MARTINI2On) Forms the $L_{\beta^{\prime}}$, but Not $P_{\beta^{\prime}}$, Phase. DPPC, with its large headgroup and strong head-head interactions, favors a tilted gel $\left(\mathrm{L}_{\beta^{\prime}}\right)$ phase at lower temperatures. Therefore, during the $\mathrm{L}_{\alpha} \rightarrow \mathrm{L}_{\beta^{\prime}}$ transition upon simulated cooling, the tilted ripple $\left(\mathrm{P}_{\beta^{\prime}}\right)$ state occurs. Lipids exhibiting an $\mathrm{L}_{\beta}$ state at lower temperatures, such as phosphatidylethanolamines, ${ }^{75}$ show a single-phase transition and do not form the ripple phase. ${ }^{20}$ In our small membrane simulations, MARTINI2n (Figure S2) and MARTINI3a/b (Figures S4 and S5) mostly froze into $\mathrm{L}_{\beta}$ states below transition temperatures, in accordance with previous studies. ${ }^{36,49}$ MARTINI2n was previously shown to sample the $\mathrm{L}_{\beta^{\prime}}$ phase only upon enhanced sampling. ${ }^{76}$ The frozen lipids in the upper leaflet of replicate 2 of MARTINI3a (Figure S4) are weakly tilted, suggesting that this FF may also capture the $\mathrm{L}_{\beta^{\prime}}$ phase upon enhanced sampling. This propensity to freeze into the $\mathrm{L}_{\beta}$ state may underlie the inability of these FFs to capture the $\mathrm{P}_{\beta^{\prime}}$ phase. However, MARTINI2On, possessing optimized DPPC bond parameters that closely match macroscopic membrane properties from allatom simulations, spontaneously captured the $\mathrm{L}_{\beta^{\prime}}$ phase in many of the small membrane replicates upon cooling with classical MD simulations (Figure S3). This agrees with a previous study ${ }^{31}$ that developed the MARTINI2On DPPC parameters, where the authors also observed the formation of the $L_{\beta^{\prime}}$ phase without enhanced sampling. Even in replicates that formed interdigitated ripple-like configurations (Figure S3), the noninterdigitated gel-like domains often resembled the $\mathrm{L}_{\beta^{\prime}}$ phase.

To further contrast the lipid tilt in the gel phase configurations of DPPC MARTINI2n and MARTINI2On membranes, we chose representative replicates (\#7 in Figure S2, \#3 and \#7 in Figure S3) that had frozen into gel states after SAMD without sampling interdigitated ripple-like states, and further equilibrated them at $280 \mathrm{~K}$ for $5 \mu \mathrm{s}$ in the NPT ensemble. The final MD snapshots for MARTINI2n (\#7 from Figure S2) and MARTINI2On (\#7 from Figure S3) are illustrated in Figure 6a,b. Clearly, MARTINI2n and MARTINI2On form stable $\mathrm{L}_{\beta}$ and $\mathrm{L}_{\beta^{\prime}}$ configurations, respectively, at $280 \mathrm{~K}$. For both systems, we quantified the extent of lipid tilt from the tilt angle $\left(\theta^{\circ}\right)$ distributions of lipids with their respective leaflet normal (Methods; Figure 6c). The MARTINI2n and MARTINI2On gel phase tilt distributions were observed to have modes at 7.3 and $15.2^{\circ}$, respectively.

To ensure that the MARTINI2On $\mathrm{L}_{\beta^{\prime}}$ configuration was not a consequence of finite size artifacts, we created a six time larger membrane by repeating the membrane from Figure $6 \mathrm{~b}$ across periodic boundaries ( $3 x$ times $2 y$; similar to Figure 5). This initial configuration is shown in Figure $6 \mathrm{~d}$, and was simulated at $280 \mathrm{~K}$ for $5 \mu \mathrm{s}$. From the final snapshot (Figure $6 \mathrm{~d}$ ), the $\mathrm{L}_{\beta^{\prime}}$ configuration is completely preserved. Additionally, simulated cooling of a large $\mathrm{L}_{\alpha}$ MARTINI2On membrane from 350 to $280 \mathrm{~K}$ also resulted in a $\mathrm{L}_{\beta^{\prime}}$ configuration (Figure $4 \mathrm{~b}$ ). These observations strongly suggest that MARTINI2On membranes can sample the true $\mathrm{L}_{\beta^{\prime}}$ phase at lower temperatures, without any finite size artifacts, and in agreement with previous studies. ${ }^{31}$ However, our multireplicate simulations show that MARTINI2On membranes also stochastically sample the $L_{\beta}$ phase, as shown by replicates \#2 and \#8 in smaller membranes (Figure S3), and from the simulations of large membranes (Figures 5b, S10 and S11). Therefore, there may be room for further optimization of the MARTINI2On DPPC FF for accurate reproduction of low-temperature phase behavior.

Interestingly, when replicate \#3 from Figure S3 was equilibrated for $5 \mu \mathrm{s}$ at $280 \mathrm{~K}$, it showed the formation of a weakly interdigitated titled domain (Figure S13a), a potential precursor for the formation of a true ripple phase. To assess this further, we set up a six-times larger membrane (Figure 

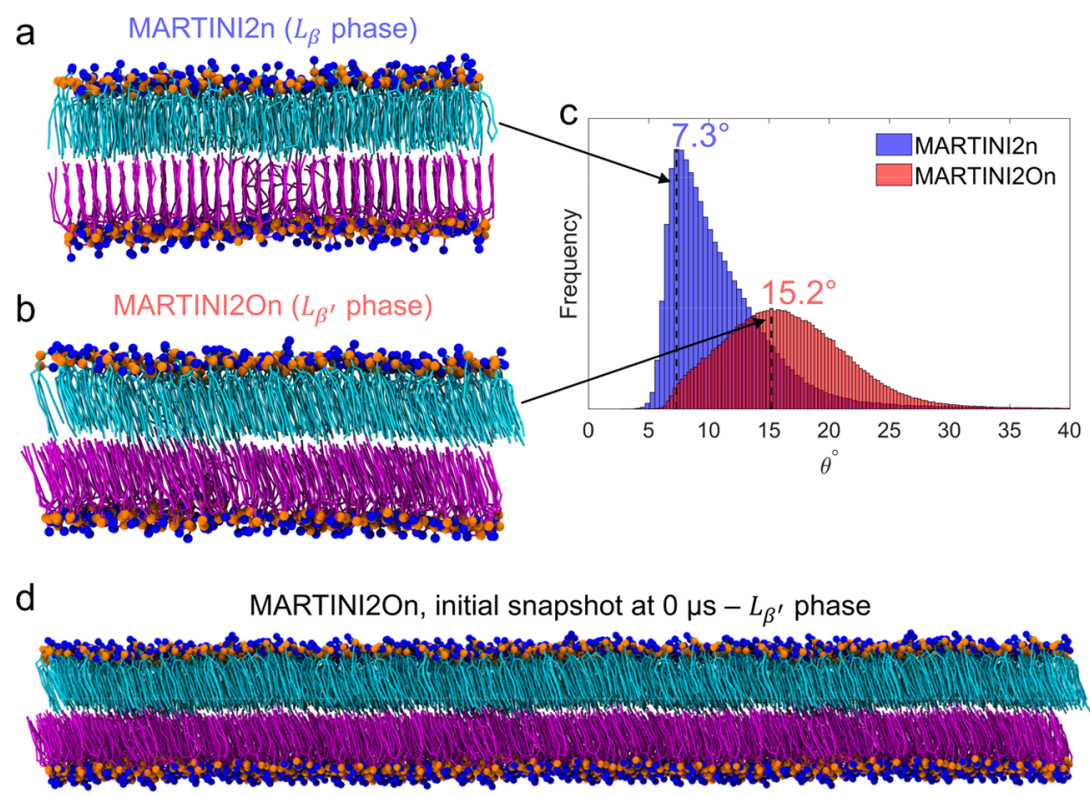

Final snapshot after $5 \mu$ s equilibrium MD at $280 \mathrm{~K}-L_{\beta^{\prime}}$ phase

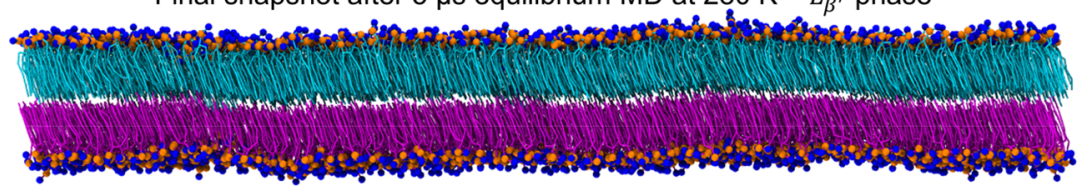

Figure 6. Comparison of gel-phase structures of MARTINI membranes. Gel-phase DPPC bilayer structures with (a) standard MARTINI2n FF and (b) optimized MARTINI2On FF are shown. MARTINI2n membranes freeze into the straight gel-phase $\left(\mathrm{L}_{\beta}\right)$, whereas MARTINI2On membranes often sample the tilted gel phase $\left(\mathrm{L}_{\beta^{\prime}}\right)$, quantified by the lipid tilt-angle distributions $(\mathrm{c})$. (d) To confirm that this was a true thermodynamic phase and not a finite size effect, the smaller $\mathrm{L}_{\beta^{\prime}}$ membrane in (a) was replicated $6 x$ (initial snapshot) and then equilibrated for $5 \mu \mathrm{s}$ at $280 \mathrm{~K}$. The membrane remained in the $\mathrm{L}_{\beta^{\prime}}$ phase thus ruling out finite size artifacts.

S13b) by repeating the smaller system across periodic boundaries along the membrane plane (similar to Figure 5a). After $10 \mu \mathrm{s}$ of constant temperature EQMD at $280 \mathrm{~K}$, the interdigitation almost completely disappeared as shown by the final MD snapshot (Figure S13b) and corresponding Voronoithickness maps (Figure S13c) indicating that this replicate was unable to sample the ripple phase.

\section{CONCLUSIONS}

Molecular simulations at various levels of FF descriptions, ranging from all-atom and united atom models at the finer scales to DPD and MARTINI models at higher levels of coarse graining, have been extensively used to study the rich variety of equilibrium phases observed in model phospholipid bilayer membranes. ${ }^{1,17,77}$ In particular, the rippled $\mathrm{P}_{\beta^{\prime}}$ phase, sensitive to initial configurations, annealing protocols, and finite-size effects, has been challenging to capture using MD simulations. ${ }^{20,25,36,43}$ Although all-atom FFs have successfully captured the formation of the ripple phase (Table 1), the ability of the widely used MARTINI FFs to stabilize this intermediate phase, which occurs below the main gel-to-liquid crystalline transition, has been a matter of some debate. ${ }^{36,49}$

In this article, we assessed the ability of MARTINI DPPC lipid membranes described by six MARTINI FF variants (see Introduction and Methods) toward capturing the ripple phase. Previous experiments ${ }^{35,39-42}$ and all-atom simulations ${ }^{19,31,45,46}$ have shown that DPPC membranes sample the tilted ripple phase at pretransition temperatures. Previous MARTINI studies yielded conflicting observations, with some inferring that MARTINI DPPC lipid membranes could not capture the ripple state, ${ }^{36}$ while other studies observed otherwise. ${ }^{31,48,49}$ Therefore, we established a standard simulated annealingbased MD protocol, built on insights from previous studies, $^{18-20,31,45,46}$ for reliably producing the tilted ripple phase in multiple all-atom DPPC membrane MD replicates by cooling from the fluid phase to below the phase transition, followed by constant temperature equilibration. The structure of these rippled membranes resembled those from previous studies. ${ }^{19,20,45,46}$ We then applied the same MD protocol to fluid-phase MARTINI DPPC membranes with six MARTINI FF variants and 10 independent MD replicates per variant. Simulations with all the MARTINI FFs showed that a fraction of replicates formed interdigitated ripple-like states. These simulations also reinforced the importance of using multiple independent MD replicates for robustly observing the phase behavior. ${ }^{18,52}$ The lateral orientation of interdigitated domains in these ripple-like membranes was different compared to those from all-atom simulations and resembled a membrane with coexisting interdigitated $\mathrm{L}_{\alpha}$ and gel phases.

Next, we explored whether the MARTINI ripple-like membranes were kinetically trapped states enforced by finite system size effects. To this end, we first re-established the allatom protocols by employing the aforementioned MD cooling protocol on large all-atom DPPC membranes. We observed that these larger membranes also formed the tilted ripple phase with reticulated interdigitated domains, in agreement with previously reported large-membrane all-atom DPPC simulations. $^{31,45}$ However, when similarly large MARTINI DPPC membranes (four FF variants and two replicates each) were cooled from the fluid phase, they failed to capture ripple-like 
states and froze directly into the gel phase. To confirm this behavior via an orthogonal approach, we created larger membranes from previously formed smaller ripple-like membranes, by repeating them across periodic boundaries. These larger MARTINI periodic ripple-like membranes (4 FF variants), with an initial configuration bias toward the ripple phase, were then simulated at a constant temperature of $280 \mathrm{~K}$, below the transition temperature. Strikingly, all the membranes showed a progressive loss of the interdigitated ripple-like domains, and either transitioned, or were in the process of transitioning, into the gel phase. Based on these findings, we attribute the ripple-like states observed in the smaller MARTINI membranes to finite size artifacts. In the case of the MARTINI2On DPPC FF, the inability to form the tilted ripple was despite its ability to capture the tilted gel phase at lower temperatures without the need for enhanced sampling.

In summary, our systematic observations suggest that the current MARTINI DPPC FFs, including the recently developed MARTINI3, may not be able to capture the $\mathrm{P}_{\beta^{\prime}}$ phase, which involves an intricate interplay of lipid tail interdigitation, tilt, and headgroup hydration in the ripple state of the membrane. CG force fields such as MARTINI are known to overestimate enthalpy to compensate for an intrinsically low entropy due to reduced degrees of freedom. ${ }^{78}$ Specifically reparametrizing the MARTINI FFs to correct this enthalpy-entropy imbalance and better reproduce membrane thermodynamics as well as phase behavior is expected to yield improved CG models that may bridge the qualitative and quantitative gap toward all-atom MD simulations, while unlocking the speed and scaling advantages associated with the versatile MARTINI framework.

\section{ASSOCIATED CONTENT}

\section{SI Supporting Information}

The Supporting Information is available free of charge at https://pubs.acs.org/doi/10.1021/acs.jpcb.1c03277.

All-atom MD simulation replicate reproducing the ripple phase; 10 independent cooling MD simulation replicates with the six MARTINI FFs; and large all-atom and MARTINI membrane simulations (PDF)

\section{AUTHOR INFORMATION}

\section{Corresponding Author}

K. Ganapathy Ayappa - Department of Chemical Engineering, Indian Institute of Science, Bengaluru 560012, India; Centre for BioSystems Science and Engineering, Indian Institute of Science, Bengaluru 560012, India; 이이.org/0000-00017599-794X; Email: ayappa@iisc.ac.in

\section{Authors}

Pradyumn Sharma - Department of Chemical Engineering, Indian Institute of Science, Bengaluru 560012, India; (1) orcid.org/0000-0002-4489-1832

Rajat Desikan - Department of Chemical Engineering, Indian Institute of Science, Bengaluru 560012, India; Present Address: Certara QSP, Certara UK Limited, Sheffield,

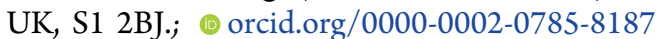

Complete contact information is available at:

https://pubs.acs.org/10.1021/acs.jpcb.1c03277

\section{Author Contributions}

${ }^{\S}$ P.S. and R.D. co-first authors.

\section{Notes}

The authors declare no competing financial interest.

\section{ACKNOWLEDGMENTS}

We thank the Supercomputer Education and Research Center (SERC) computational facility at the Indian Institute of Science, Bengaluru, for access to supercomputing resources. K.G.A. acknowledges funding by a grant from the Department of Science and Technology, Government of India.

\section{REFERENCES}

(1) Kulkarni, C. V. Lipid Crystallization: From Self-Assembly to Hierarchical and Biological Ordering. Nanoscale 2012, 4, 5779-5791.

(2) Nagle, J. F. Theory of the Main Lipid Bilayer Phase Transition. Ann. Rev. Phys. Chem. 1980, 31, 157-196.

(3) Harayama, T.; Riezman, H. Understanding the Diversity of Membrane Lipid Composition. Nat. Rev. Mol. Cell Biol. 2018, 19, 281-296.

(4) Kohli, A. G.; Kierstead, P. H.; Venditto, V. J.; Walsh, C. L.; Szoka, F. C. Designer Lipids for Drug Delivery: From Heads to Tails. J. Controlled Release 2014, 190, 274-287.

(5) Anderson, M.; Omri, A. The Effect of Different Lipid Components on the in Vitro Stability and Release Kinetics of Liposome Formulations. Drug Delivery 2004, 11, 33-39.

(6) Kulkarni, A.; Chandrasekar, V.; Natarajan, S. K.; Ramesh, A.; Pandey, P.; Nirgud, J.; Bhatnagar, H.; Ashok, D.; Ajay, A. K.; Sengupta, S. A Designer Self-Assembled Supramolecule Amplifies Macrophage Immune Responses against Aggressive Cancer. Nat. Biomed. Eng. 2018, 2, 589-599.

(7) Phillips, R.; Ursell, T.; Wiggins, P.; Sens, P. Emerging Roles for Lipids in Shaping Membrane-Protein Function. Nature 2009, 459, 379-385.

(8) Blicher, A.; Wodzinska, K.; Fidorra, M.; Winterhalter, M.; Heimburg, T. The Temperature Dependence of Lipid Membrane Permeability, Its Quantized Nature, and the Influence of Anesthetics. Biophys. J. 2009, 96, 4581-4591.

(9) Sharma, P.; Parthasarathi, S.; Patil, N.; Waskar, M.; Raut, J. S.; Puranik, M.; Ayappa, K. G.; Basu, J. K. Assessing Barriers for Antimicrobial Penetration in Complex Asymmetric Bacterial Membranes: A Case Study with Thymol. Langmuir 2020, 36, 8800-8814.

(10) Pérez-Isidoro, R.; Sierra-Valdez, F. J.; Ruiz-Suárez, J. C. Anesthetic Diffusion through Lipid Membranes Depends on the Protonation Rate. Sci. Rep. 2014, 4, 7534.

(11) Yilmaz, N.; Kobayashi, T. Assemblies of Pore-Forming Toxins Visualized by Atomic Force Microscopy. Biochim. Biophys. Acta 2016, 1858, 500-511.

(12) Rojko, N.; Anderluh, G. How Lipid Membranes Affect Pore Forming Toxin Activity. Acc. Chem. Res. 2015, 48, 3073-3079.

(13) Gerelli, Y.; Porcar, L.; Lombardi, L.; Fragneto, G. Lipid Exchange and Flip-Flop in Solid Supported Bilayers. Langmuir 2013, 29, 12762-12769.

(14) Pomorski, T. G.; Menon, A. K. Lipid Somersaults: Uncovering the Mechanisms of Protein-Mediated Lipid Flipping. Prog. Lipid Res. 2016, 64, 69-84.

(15) Andersen, S. S. L.; Jackson, A. D.; Heimburg, T. Towards a Thermodynamic Theory of Nerve Pulse Propagation. Prog. Neurobiol. 2009, 88, 104-113.

(16) de Smet, M.; Langereis, S.; van den Bosch, S.; Grüll, H. Temperature-Sensitive Liposomes for Doxorubicin Delivery under MRI Guidance. J. Controlled Release 2010, 143, 120-127.

(17) Enkavi, G.; Javanainen, M.; Kulig, W.; Róg, T.; Vattulainen, I. Multiscale Simulations of Biological Membranes: The Challenge To Understand Biological Phenomena in a Living Substance. Chem. Rev. 2019, 119, 5607-5774.

(18) Sun, L.; Böckmann, R. A. Membrane Phase Transition during Heating and Cooling: Molecular Insight into Reversible Melting. Eur. Biophys. J. 2018, 47, 151-164. 
(19) de Vries, A. H.; Yefimov, S.; Mark, A. E.; Marrink, S. J. Molecular Structure of the Lecithin Ripple Phase. Proc. Natl. Acad. Sci. U.S.A. 2005, 102, 5392-5396.

(20) Lenz, O.; Schmid, F. Structure of Symmetric and Asymmetric "Ripple" Phases in Lipid Bilayers. Phys. Rev. Lett. 2007, 98, 058104.

(21) Kranenburg, M.; Smit, B. Phase Behavior of Model Lipid Bilayers. J. Phys. Chem. B 2005, 109, 6553-6563.

(22) Marrink, S. J.; Tieleman, D. P. Perspective on the Martini Model. Chem. Soc. Rev. 2013, 42, 6801-6822.

(23) Marrink, S. J.; Risselada, H. J.; Yefimov, S.; Tieleman, D. P.; de Vries, A. H. The MARTINI Force Field: Coarse Grained Model for Biomolecular Simulations. J. Phys. Chem. B 2007, 111, 7812-7824.

(24) Bruininks, B. M. H.; Souza, P. C. T.; Marrink, S. J. A Practical View of the Martini Force Field. In Biomolecular Simulations: Methods and Protocols; Bonomi, M., Camilloni, C., Eds.; Methods in Molecular Biology; Springer: New York, NY, 2019, pp 105-127.

(25) Srivastava, A.; Debnath, A. Asymmetry and Rippling in Mixed Surfactant Bilayers from All-Atom and Coarse-Grained Simulations: Interdigitation and Per Chain Entropy. J. Phys. Chem. B 2020, 124, 6420-6436.

(26) Vaiwala, R.; Sharma, P.; Puranik, M.; Ayappa, K. G. Developing a Coarse-Grained Model for Bacterial Cell Walls: Evaluating Mechanical Properties and Free Energy Barriers. J. Chem. Theory Comput. 2020, 16, 5369-5384.

(27) Ingólfsson, H. I.; Melo, M. N.; van Eerden, F. J.; Arnarez, C.; Lopez, C. A.; Wassenaar, T. A.; Periole, X.; de Vries, A. H.; Tieleman, D. P.; Marrink, S. J. Lipid Organization of the Plasma Membrane. J. Am. Chem. Soc. 2014, 136, 14554-14559.

(28) Alessandri, R.; Souza, P. C. T.; Thallmair, S.; Melo, M. N.; de Vries, A. H.; Marrink, S. J. Pitfalls of the Martini Model. J. Chem. Theory Comput. 2019, 15, 5448-5460.

(29) Desikan, R.; Patra, S. M.; Sarthak, K.; Maiti, P. K.; Ayappa, K. G. Comparison of Coarse-Grained (MARTINI) and Atomistic Molecular Dynamics Simulations of $\alpha$ and $\beta$ Toxin Nanopores in Lipid Membranes. J. Chem. Sci. 2017, 129, 1017-1030.

(30) Desikan, R.; Behera, A.; Maiti, P. K.; Ayappa, K. G. Chapter Sixteen-Using Multiscale Molecular Dynamics Simulations to Obtain Insights into Pore Forming Toxin Mechanisms. In Methods in Enzymology; Heuck, A. P., Ed.; Pore-Forming Toxins; Academic Press, 2021; Vol. 649, pp 461-502.

(31) Carpenter, T. S.; López, C. A.; Neale, C.; Montour, C.; Ingólfsson, H. I.; Di Natale, F.; Lightstone, F. C.; Gnanakaran, S. Capturing Phase Behavior of Ternary Lipid Mixtures with a Refined Martini Coarse-Grained Force Field. J. Chem. Theory Comput. 2018, $14,6050-6062$.

(32) Globisch, C.; Krishnamani, V.; Deserno, M.; Peter, C. Optimization of an Elastic Network Augmented Coarse Grained Model to Study CCMV Capsid Deformation. PLoS One 2013, 8, No. e60582.

(33) Souza, P. C. T.; Alessandri, R.; Barnoud, J.; Thallmair, S.; Faustino, I.; Grünewald, F.; Patmanidis, I.; Abdizadeh, H.; Bruininks, B. M. H.; Wassenaar, T. A.; et al. Martini 3: A General Purpose Force Field for Coarse-Grained Molecular Dynamics. Nat. Methods 2021, $18,382-388$.

(34) Biltonen, R. L.; Lichtenberg, D. The Use of Differential Scanning Calorimetry as a Tool to Characterize Liposome Preparations. Chem. Phys. Lipids 1993, 64, 129-142.

(35) Tardieu, A.; Luzzati, V.; Reman, F. C. Structure and Polymorphism of the Hydrocarbon Chains of Lipids: A Study of Lecithin-Water Phases. J. Mol. Biol. 1973, 75, 711-733.

(36) Rodgers, J. M.; Sørensen, J.; de Meyer, F. J.-M.; Schiøtt, B.; Smit, B. Understanding the Phase Behavior of Coarse-Grained Model Lipid Bilayers through Computational Calorimetry. J. Phys. Chem. B 2012, 116, 1551-1569.

(37) Riske, K. A.; Barroso, R. P.; Vequi-Suplicy, C. C.; Germano, R.; Henriques, V. B.; Lamy, M. T. Lipid Bilayer Pre-Transition as the Beginning of the Melting Process. Biochim. Biophys. Acta, Biomembr. 2009, 1788, 954-963.
(38) Heimburg, T. A Model for the Lipid Pretransition: Coupling of Ripple Formation with the Chain-Melting Transition. Biophys. J. 2000, 78, 1154-1165.

(39) Sun, W. J.; Tristram-Nagle, S.; Suter, R. M.; Nagle, J. F. Structure of the Ripple Phase in Lecithin Bilayers. Proc. Natl. Acad. Sci. U.S.A. 1996, 93, 7008-7012.

(40) Katsaras, J.; Tristram-Nagle, S.; Liu, Y.; Headrick, R. L.; Fontes, E.; Mason, P. C.; Nagle, J. F. Clarification of the Ripple Phase of Lecithin Bilayers Using Fully Hydrated, Aligned Samples. Phys. Rev. E: Stat. Phys., Plasmas, Fluids, Relat. Interdiscip. Top. 2000, 61, 56685677.

(41) Sengupta, K.; Raghunathan, V. A.; Katsaras, J. Structure of the Ripple Phase of Phospholipid Multibilayers. Phys. Rev. E: Stat. Phys., Plasmas, Fluids, Relat. Interdiscip. Top. 2003, 68, 031710.

(42) Kamal, M. A.; Raghunathan, V. A. Naphthalene Derivatives Induce Acyl Chain Interdigitation in Dipalmitoylphosphatidylcholine Bilayers. J. Phys. Chem. B 2016, 120, 164-172.

(43) Debnath, A.; Thakkar, F. M.; Maiti, P. K.; Kumaran, V.; Ayappa, K. G. Laterally Structured Ripple and Square Phases with One and Two Dimensional Thickness Modulations in a Model Bilayer System. Soft Matter 2014, 10, 7630-7637.

(44) Pluhackova, K.; Kirsch, S. A.; Han, J.; Sun, L.; Jiang, Z.; Unruh, T.; Böckmann, R. A. A Critical Comparison of Biomembrane Force Fields: Structure and Dynamics of Model DMPC, POPC, and POPE Bilayers. J. Phys. Chem. B 2016, 120, 3888-3903.

(45) Kirsch, S. A.; Böckmann, R. A. Coupling of Membrane Nanodomain Formation and Enhanced Electroporation near Phase Transition. Biophys. J. 2019, 116, 2131-2148.

(46) Khakbaz, P.; Klauda, J. B. Investigation of Phase Transitions of Saturated Phosphocholine Lipid Bilayers via Molecular Dynamics Simulations. Biochim. Biophys. Acta, Biomembr. 2018, 1860, 14891501.

(47) Debnath, A.; Ayappa, K. G.; Kumaran, V.; Maiti, P. K. The Influence of Bilayer Composition on the Gel to Liquid Crystalline Transition. J. Phys. Chem. B 2009, 113, 10660-10668.

(48) Lin, X.; Gu, N. Surface Properties of Encapsulating Hydrophobic Nanoparticles Regulate the Main Phase Transition Temperature of Lipid Bilayers: A Simulation Study. Nano Res. 2014, 7, 11951204.

(49) Stelter, D.; Keyes, T. Enhanced Sampling of Phase Transitions in Coarse-Grained Lipid Bilayers. J. Phys. Chem. B 2017, 121, 57705780.

(50) Yesylevskyy, S. O.; Schäfer, L. V.; Sengupta, D.; Marrink, S. J. Polarizable Water Model for the Coarse-Grained MARTINI Force Field. PLoS Comput. Biol. 2010, 6, No. e1000810.

(51) Souza, P. C. T.; Marrink, S. J. MARTINI 3-Open BetaRelease. The MARTINI web portal, 2020. http://cgmartini.nl.

(52) Grossfield, A.; Patrone, P. N.; Roe, D. R.; Schultz, A. J.; Siderius, D.; Zuckerman, D. M. Best Practices for Quantification of Uncertainty and Sampling Quality in Molecular Simulations [Article v1.0]. Living J. Comp. Mol. Sci. 2019, 1, 5067.

(53) Jo, S.; Kim, T.; Iyer, V. G.; Im, W. CHARMM-GUI: A WebBased Graphical User Interface for CHARMM. J. Comput. Chem. 2008, 29, 1859-1865.

(54) Lee, J.; Cheng, X.; Swails, J. M.; Yeom, M. S.; Eastman, P. K.; Lemkul, J. A.; Wei, S.; Buckner, J.; Jeong, J. C.; Qi, Y.; et al. CHARMM-GUI Input Generator for NAMD, GROMACS, AMBER, OpenMM, and CHARMM/OpenMM Simulations Using the CHARMM36 Additive Force Field. J. Chem. Theory Comput. 2016, $12,405-413$.

(55) Wu, E. L.; Cheng, X.; Jo, S.; Rui, H.; Song, K. C.; DávilaContreras, E. M.; Qi, Y.; Lee, J.; Monje-Galvan, V.; Venable, R. M.; et al. CHARMM-GUIMembrane Buildertoward realistic biological membrane simulations. J. Comput. Chem. 2014, 35, 1997-2004.

(56) Meyer, H. W. Pretransition-Ripples in Bilayers of Dipalmitoylphosphatidylcholine: Undulation or Periodic Segments? A FreezeFracture Study. Biochim. Biophys. Acta, Lipids Lipid Metab. 1996, $1302,138-144$. 
(57) Pastor, R. W.; MacKerell, A. D. Development of the CHARMM Force Field for Lipids. J. Phys. Chem. Lett. 2011, 2, 1526-1532.

(58) Klauda, J. B.; Venable, R. M.; Freites, J. A.; O’Connor, J. W.; Tobias, D. J.; Mondragon-Ramirez, C.; Vorobyov, I.; MacKerell, A. D.; Pastor, R. W. Update of the CHARMM All-Atom Additive Force Field for Lipids: Validation on Six Lipid Types. J. Phys. Chem. B 2010, 114, 7830-7843.

(59) Abraham, M. J.; Murtola, T.; Schulz, R.; Páll, S.; Smith, J. C.; Hess, B.; Lindahl, E. GROMACS: High Performance Molecular Simulations through Multi-Level Parallelism from Laptops to Supercomputers. SoftwareX 2015, 1-2, 19-25.

(60) Braun, E.; Gilmer, J.; Mayes, H. B.; Mobley, D. L.; Monroe, J. I.; Prasad, S.; Zuckerman, D. M. Best Practices for Foundations in Molecular Simulations [Article v1.0]. Living J. Comp. Mol. Sci. 2019, 1, 5957.

(61) Smith, D. J.; Klauda, J. B.; Sodt, A. J. Simulation Best Practices for Lipid Membranes [Article v1.0]. Living J. Comp. Mol. Sci. 2019, 1, 5966.

(62) Lemkul, J. From Proteins to Perturbed Hamiltonians: A Suite of Tutorials for the GROMACS-2018 Molecular Simulation Package [Article v1.0]. Living J. Comp. Mol. Sci. 2019, 1, 5068.

(63) Darden, T.; York, D.; Pedersen, L. Particle mesh Ewald: AnN. $\log (\mathrm{N})$ method for Ewald sums in large systems. J. Chem. Phys. 1993, 98, 10089-10092.

(64) Hess, B. P-LINCS: A Parallel Linear Constraint Solver for Molecular Simulation. J. Chem. Theory Comput. 2008, 4, 116-122.

(65) Bussi, G.; Donadio, D.; Parrinello, M. Canonical Sampling through Velocity Rescaling. J. Chem. Phys. 2007, 126, 014101.

(66) Parrinello, M.; Rahman, A. Polymorphic Transitions in Single Crystals: A New Molecular Dynamics Method. J. Appl. Phys. 1981, 52, 7182-7190.

(67) Alavi, S.; Thompson, D. L. Molecular Dynamics Studies of Melting and Some Liquid-State Properties of 1-Ethyl-3-Methylimidazolium Hexafluorophosphate [Emim][PF6]. J. Chem. Phys. 2005, 122, 154704.

(68) Zhang, Y.; Maginn, E. J. A Comparison of Methods for Melting Point Calculation Using Molecular Dynamics Simulations. J. Chem. Phys. 2012, 136, 144116.

(69) Wassenaar, T. A.; Ingólfsson, H. I.; Böckmann, R. A.; Tieleman, D. P.; Marrink, S. J. Computational Lipidomics with Insane: A Versatile Tool for Generating Custom Membranes for Molecular Simulations. J. Chem. Theory Comput. 2015, 11, 2144-2155.

(70) de Jong, D. H.; Baoukina, S.; Ingólfsson, H. I.; Marrink, S. J. Martini Straight: Boosting Performance Using a Shorter Cutoff and GPUs. Comput. Phys. Commun. 2016, 199, 1-7.

(71) Tironi, I. G.; Sperb, R.; Smith, P. E.; van Gunsteren, W. F. A Generalized Reaction Field Method for Molecular Dynamics Simulations. J. Chem. Phys. 1995, 102, 5451-5459.

(72) Humphrey, W.; Dalke, A.; Schulten, K. VMD: Visual Molecular Dynamics. J. Mol. Graphics 1996, 14, 33-38.

(73) Guixà-González, R.; Rodriguez-Espigares, I.; Ramírez-Anguita, J. M.; Carrió-Gaspar, P.; Martinez-Seara, H.; Giorgino, T.; Selent, J. MEMBPLUGIN: Studying Membrane Complexity in VMD. Bioinformatics 2014, 30, 1478-1480.

(74) Varadarajan, V.; Desikan, R.; Ayappa, K. G. Assessing the Extent of the Structural and Dynamic Modulation of Membrane Lipids Due to Pore Forming Toxins: Insights from Molecular Dynamics Simulations. Soft Matter 2020, 16, 4840-4857.

(75) Koynova, R.; Caffrey, M. Phases and Phase Transitions of the Hydrated Phosphatidylethanolamines. Chem. Phys. Lipids 1994, 69, $1-34$.

(76) Nagai, T.; Okamoto, Y. Replica-Exchange Molecular Dynamics Simulation of a Lipid Bilayer System with a Coarse-Grained Model. Mol. Simul. 2012, 38, 437-441.

(77) Manna, M.; Nieminen, T.; Vattulainen, I. Understanding the Role of Lipids in Signaling Through Atomistic and Multiscale Simulations of Cell Membranes. Annu. Rev. Biophys. 2019, 48, 421439.
(78) Jarin, Z.; Newhouse, J.; Voth, G. A. Coarse-Grained Force Fields from the Perspective of Statistical Mechanics: Better Understanding of the Origins of a MARTINI Hangover. J. Chem. Theory Comput. 2021, 17, 1170-1180. 